Edinburgh Anisotropy Project

xyl05gp_rev.doc

11 April 2006

\title{
Converted-wave imaging in anisotropic media: Theory and case studies*
}

\author{
Xiang-Yang $\mathrm{Li}^{1}$, Hengchang Dai ${ }^{1}$ and Fabio Mancini ${ }^{2}$ \\ ${ }^{1}$ British Geological Survey, West Mains Road, Edinburgh EH9 3LA, UK \\ ${ }^{2}$ Total E\&P UK PLC, Crawpeel Road, Altens Industrial Estate, Aberdeen, AB12 3FG \\ * Paper presented in the EAGE/SEG research workshop on multicomponent seismic, \\ 5-8 September 2005, Pau, France \\ Suggested short title: Converted-wave imaging in anisotropic media \\ Intended publication: Geophysical Prospecting \\ Submitted on $\quad 14$ December 2005 \\ Revised on $\quad 11$ April 2006
}

\footnotetext{
*Address for correspondence

Xiang-Yang Li

Britigh Geological Survey

Murchison House,

West Mains Road

Edinburgh EH9 3LA

Scotland,UK

xyl@bgs.ac.uk
} 


\section{SUMMARY}

Common-conversion-point binning associated with converted-wave ( $C$-wave) processing complicates the task of parameter estimation, especially in anisotropic media. To overcome this problem, we derive new expressions for converted-wave prestack time migration (PSTM) in anisotropic media and illustrate their applications using both 2D and 3D data examples.

The converted-wave kinematic response in inhomogeneous media with vertical transverse isotropy (VTI) is separated into two parts: the response in horizontally layered VTI media and the response from a point scatter. The former controls the stacking process and the latter controls the process of PSTM. The $C$-wave traveltime in horizontally layered VTI media is determined by four parameters: the $C$-wave stacking velocity $V_{\mathrm{C} 2}$, the vertical and effective velocity ratios $\gamma_{0}$ and $\gamma_{\text {eff }}$, and the $C$-wave anisotropic parameter $\chi_{\text {eff. }}$ These four parameters are referred to as the $C$-wave stacking velocity model. In contrast, the $C$-wave diffraction time from a point scatter is determined by five parameters: $\gamma_{0}, V_{\mathrm{P} 2}, V_{\mathrm{S} 2}, \eta_{\mathrm{eff}}$ and $\zeta_{\mathrm{eff}}$, where $\eta_{\mathrm{eff}}$ and $\zeta_{\text {eff }}$ are, respectively, the $P$ - and $S$-wave anisotropic parameters, and $V_{\mathrm{P} 2}$ and $V_{\mathrm{S} 2}$ are the corresponding stacking velocities. $V_{\mathrm{P} 2}, V_{\mathrm{S} 2}, \eta_{\text {eff }}$ and $\zeta_{\text {eff }}$ are referred to as the $C$-wave PSTM velocity model. There is a one-to-one analytical link between the stacking velocity model and the PSTM velocity model. There is also a simple analytic link between the C-wave stacking velocities $V_{\mathrm{C} 2}$ and the migration velocity $V_{\mathrm{Cmig}}$, which is in turn linked to $V_{\mathrm{P} 2}$ and $V_{\mathrm{S} 2}$.

Based on the above, we have developed an interactive processing scheme to build the stacking and PSTM velocity model and to perform 2D and 3D $C$-wave anisotropic PSTM. Real data applications show that the PSTM scheme substantially improves the quality of $C$ wave imaging compared with the DMO (dip moveout) scheme, and these improvements have been confirmed by drilling.

\section{INTRODUCTION}

Converted-wave exploration started in the 1970's. Since then, a considerable amount of effort has been devoted to develop workflows for processing converted-wave data. Stewart et al. (2002) gave a good overview on these efforts. However, unlike P-waves, there are still no recognized standard processing flows for converted-waves because of their asymmetric raypaths and sensitivity to anisotropy. This paper reviews the recent developments in converted-wave processing for vertical transverse isotropy (VTI), and addresses issues such as how many parameters are required to perform converted-wave anisotropic prestack time migration (PSTM), and how to estimate these parameters. 
A PS-converted-wave with a down-going P-wave path and up-going S-wave path is referred to as a C-wave, following Thomsen (1999). The moveout of this C-wave is inherently non-hyperbolic due to the asymmetry raypath. Another issue is the calculation of the conversion-point. Most of the early effort in converted-wave processing is devoted to solve these two problems (e.g. Tesser and Behle 1988; Zhang 1992; Harrison 1992; amongst others).

As the advent of marine $4 \mathrm{C}$ technology, the application of converted-wave in imaging beneath gas cloud and interpreting subtle straitigraphic traps attracted considerable interests and these applications have now evolved into major drivers for the further development of the converted-wave technology. However, new complications arise in converted-wave processing due to the widely occurrence of polar anisotropy (VTI in particular) in marine sediments. The presence of polar anisotropy causes mis-positioning both vertical and laterally, and affects imaging quality. These effects can no longer be neglected in converted-wave processing due to the ever-increasing need for better return. For these reasons, it is still not wholly clear what is the optimum strategy for processing converted-waves.

In $P$-wave processing, it is common to decompose the process of prestack time migration (PSTM) into CMP (common midpoint) binning, NMO (normal moveout), DMO and poststack migration. This decomposition is very useful for $P$-waves in terms of both parameter estimation and actual imaging. This is due to the fact that for $P$-waves, both the binning and DMO operations are independent of velocities (Hale 1984). Therefore, in the early development of converted-wave processing for isotropy, it was natural to develop similar schemes. The C-wave NMO and CCP (common-conversion-point) binning strategy was presented by Tessmer and Behle (1988), followed by Harrison (1992) for the DMO and post stack migration. This has been very successful until anisotropy is encountered.

Various attempts have been made to extend the DMO approach to anisotropy (e.g. Rommel, 1996; Thomsen, 1999; Tsvankin and Grechka, 2000; amongst others), hoping to repeat the previous success. However, anisotropic CCP binning and converted-wave DMO is strongly velocity-dependent and this severely limited the success of this approach. Since 1999, efforts have also been made to develop converted-wave anisotropic PSTM to replace the CCPbinning and DMO approach (e.g., Li and Druzhinin, 2000; Dai and Li 2001; Dai, 2003; amongst others). Here, we review these developments and present a unified and interactive approach for converted-wave imaging and parameter estimation in anisotropic media.

We separate the C-wave response into a zero-dip response for describing convertedwaves in horizontally-layered media, and a full-dip response for describing $\mathrm{C}$-wave scattering from a point-scatter. The former controls the common stacking process, and the latter controls 


\section{Li et al.}

the process of pre-strack time migration (PSTM). We derive analytical links between the parameters describing these two processes, since the stacking parameters are inherently linked to the imaging ones, and are not independent of each other. Initial parameter estimation is then performed during the stacking process, which forms the initial velocity model for constructing common-imaging-point (CIP) gathers and we use these gathers to update the velocity model. Both 2D and 3D data examples will be used to demonstrate the strategy.

\section{FLAT-LAYER RESPONSE AND THE STACKING VELOCITY MODEL}

Here, we derive the $C$-wave moveout signature in horizontally layered media (Figure 1), which controls the stacking process including $\mathrm{C}$-wave moveout correction and velocity analysis. Tsvankin and Thomsen (1994) first studied the C-wave zero-dip response for VTI media. More recently, Thomsen (1999) and Cheret et al. (2000) presented simplified forms. However, their simplifications are less accurate compared with the original equations in Tsvankin and Thomsen (1994). More accurate expressions are required for parameter estimation.

\section{Basic expressions}

$\mathrm{Li}$ and Yuan (2003) derived an improved four-parameter equation for quantifying the C-wave zero-dip response for VTI media:

$$
t_{C}^{2}=t_{C 0}^{2}+\frac{x^{2}}{V_{C 2}^{2}}+\frac{A_{4} x^{4}}{1+A_{5} x^{2}}
$$

where

$$
\begin{aligned}
& A_{4}=-\frac{\left(\gamma_{0} \gamma_{e f f}-1\right)^{2}+8\left(1+\gamma_{0}\right) \chi_{\text {eff }}}{4 t_{C 0}^{2} V_{C 2}^{4} \gamma_{0}\left(1+\gamma_{\text {eff }}\right)^{2}}, \text { and } \\
& A_{5}=\frac{A_{4} V_{C 2}^{2}\left(1+\gamma_{0}\right) \gamma_{\text {eff }}\left[\left(\gamma_{0}-1\right) \gamma_{\text {eff }}^{2}+2 \chi_{\text {eff }}\right]}{\left(\gamma_{0}-1\right) \gamma_{\text {eff }}^{2}\left(1-\gamma_{0} \gamma_{\text {eff }}\right)-2\left(1+\gamma_{0}\right) \gamma_{\text {eff }} \chi_{\text {eff }}} .
\end{aligned}
$$

$V_{\mathrm{C} 2}$ is the $C$-wave stacking velocity, $\gamma_{0}$ and $\gamma_{\text {eff }}$ are the vertical and effective velocity ratio, and $\chi_{\text {eff }}$ is the $C$-wave anisotropic coefficient. For the layered media in Figure 1, the first three parameters $\left(V_{\mathrm{C} 2}, \gamma_{0}\right.$ and $\left.\gamma_{\mathrm{eff}}\right)$ are defined as,

$$
\begin{aligned}
& t_{C 0} V_{C 2}^{2}=t_{P 0} V_{P 2}^{2}+t_{S 0} V_{S 2}^{2} ; t_{C 0}=t_{P 0}+t_{S 0} ; \\
& \gamma_{0}=t_{S 0} / t_{P 0} ; \\
& \gamma_{\text {eff }}=\gamma_{2}^{2} / \gamma_{0} ; \gamma_{2}=V_{P 2} / V_{S 2}
\end{aligned}
$$


where $t_{\mathrm{P} 0}$ and $t_{\mathrm{S} 0}$ are the one-way vertical $\mathrm{P}$ - and $\mathrm{S}$-wave travel time; $V_{\mathrm{P} 2}$ and $V_{\mathrm{S} 2}$ are the corresponding P- and S-wave stacking velocities; $\gamma_{2}$ is the P- and S-wave stacking velocity ratio; $t_{\mathrm{C} 0}$ is the corresponding two-way vertical C-wave traveltime.

The anisotropic coefficient $\chi_{\text {eff }}$ is defined as,

$$
\chi_{\text {eff }}=\eta_{\text {eff }} \gamma_{\text {eff }}^{2} \gamma_{0}-\zeta_{\text {eff }},
$$

where $\eta_{\text {eff }}$ is the effective P-wave anisotropic coefficient defined by Alkhalifah and Tsvankin (1995), and $\zeta_{\text {eff }}$ is the corresponding S-wave anisotropic coefficient defined by $\mathrm{Li}$ and Yuan (2003). For a stack of $n$ VTI layers, $\eta_{\text {eff }}$ and $\zeta_{\text {eff }}$ are defined as,

$$
\begin{aligned}
\eta_{e f f} & =\frac{1}{8 t_{P 0} V_{P 2}^{4}}\left[\sum_{i=1}^{n} V_{P 2 i}^{4} \Delta t_{P 0 i}\left(1+8 \eta_{i}\right)-t_{P 0} V_{P 2}^{4}\right], \text { and } \\
\zeta_{\text {eff }} & =\frac{-1}{8 t_{S 0} V_{S 2}^{4}}\left[\sum_{i=1}^{n} V_{S 2 i}^{4} \Delta t_{S 0 i}\left(1-8 \zeta_{i}\right)-t_{S 0} V_{S 2}^{4}\right] .
\end{aligned}
$$

Subscript $i$ denotes interval quantities. For a single VTI layer (e.g. the $i$-th layer), $\eta_{i}$ and $\zeta_{\mathrm{i}}$ have the following relationship with the Thomsen parameters $\varepsilon_{i}$ and $\delta_{i}$ :

$$
\eta_{i}=\left(\varepsilon_{i}-\delta_{i}\right) /\left(1+2 \delta_{i}\right) ; \quad \zeta_{i}=\eta_{i} \gamma_{2 i}^{2} / \gamma_{0 i}=\eta_{i} \gamma_{\text {effi }}^{2} .
$$

Equations (1) and (2) are controlled by four parameters $\left(V_{\mathrm{C} 2}, \gamma_{0}, \gamma_{\mathrm{eff}}\right.$ and $\left.\chi_{\mathrm{eff}}\right)$, and they are accurate for offset-depth ratio of $2.0(x / z \leq 2.0)$ ( $\mathrm{Li}$ and Yuan, 2003). These four parameters are referred to as the $\mathrm{C}$-wave stacking velocity model.

\section{Workflow for estimating the stacking velocity model}

There are four parameters in the stacking velocity model. The key questions are: how sensitive the moveout signature is to variations of these parameters; how many of these parameters we can recover from the moveout signature, and how to recover them.

According to Li and Yuan (2003), the moveout signature is not very sensitive to the variation of the $\gamma_{0}$. Consequently, $\gamma_{0}$ cannot be reliably obtained from the moveout signature. In the absence of borehole data, $\gamma_{0}$ may be obtained by a correlation of the $\mathrm{P}$ - and $\mathrm{C}$-wave stacked sections. This requires the generation of brute stack sections of both $\mathrm{P}$ - and $\mathrm{C}$-waves using the near offset data. Note that common ACP (Asymptotic Conversion Point) binning and hyperbolic moveout correction are often sufficiently accurate for near-offset data (Tessmer and Behle 1988). 


\section{Li et al.}

Once $\gamma_{0}$ is determined, the other three parameters $\left(V_{\mathrm{C} 2}, \gamma_{\text {eff }}\right.$ and $\left.\chi_{\text {eff }}\right)$ may be recovered from the moveout signature ( $\mathrm{Li} 2003$ ). This is because each of these parameters controls a particular data aperture of primary influence that can be used to invert for the corresponding parameter. $V_{\mathrm{C} 2}$ controls the near-offset hyperbolic moveout with an offset-depth ratio $(x / z)$ up to $1.0 ; \gamma_{\text {eff }}$ controls the non-hyperbolic moveout at the intermediate offset due to the asymmetric raypath with $x / z$ up to 1.5 ; $\chi_{\text {eff }}$ controls the non-hyperbolic moveout at the faroffset due to anisotropy with $x / z$ up to 2.0. Note that here we use two parameters to describe the non-hyperbolic moveout: one for the asymmetric raypath and one for anisotropy. It is possible to use just one combined parameter to describe the non-hyperbolic moveout as in Cheret et al. (2002) and Dai and Li (2005).

The above offset-dependent behaviour is illustrated in Figure 2 using a real data from the North Sea (Courtesy of Shell Expro). Figure 2a shows a common ACP (asymptotic conversion point) gather as an input; the resulted hyperbolic moveout correction using $V_{\mathrm{C} 2}$ only, ignoring the asymmetry raypath and anisotropy, is shown Figure $2 b$, and only the events at the near offset are flattened. The results of non-hyperbolic moveout correction by equation (1), accounting for the asymmetric raypath but ignoring the anisotropy (i.e. $\chi_{\text {eff }}=0$ ) are shown Figure 2c, and events up to the intermediate offsets are flattened. Only to account for both the asymmetric raypath and anisotropy are the far-offset events then satisfactorily flattened (Figure $2 \mathrm{~d})$. Accordingly, the three parameters $\left(V_{\mathrm{C} 2}, \gamma_{\mathrm{eff}}\right.$ and $\left.\chi_{\mathrm{eff}}\right)$ may be determined with sufficient accuracy and resolution by inverting the $C$-wave moveout from its corresponding data aperture (Figure 3).

Therefore, amongst the four parameters in the stacking velocity model, only three of them $\left(V_{\mathrm{C} 2}, \gamma_{\mathrm{eff}}\right.$ and $\left.\chi_{\mathrm{eff}}\right)$ may be recoverable from the $\mathrm{C}$-wave moveout signature, and parameter $\gamma_{0}$ needs to be determined from other sources of information independently. Note that, finding three parameters from moveout analysis is not trivial and may not work for all data. In such cases, additional constraints on parameter $\gamma_{\text {eff }}$ may be obtained by examining the asymmetry of the positive- and negative-offset gathers (Audebert et al. 1999; Mancini 2004), or by utilizing P-wave velocity information ( $\mathrm{Li}$ and Yuan 2003).

To sum up, a two-step work flow is needed to estimate the stacking velocity model. The first step is to determine $\gamma_{0}$ using near-offset $\mathrm{P}$ - and $\mathrm{C}$-wave brute stacks obtained by hyperbolic processing. The second step utilizes the full-offset data to determine the other three parameters using the non-hyperbolic approach defined by equations (1) and (2). During this stage, the data are sorted into common ACP gathers by

$$
x_{\text {acp }}=\frac{\gamma_{0}}{1+\gamma_{0}} x,
$$


where $x_{\text {acp }}$ is the distance between the source point and the ACP point. In this way, CCP (common-conversion point) binning is avoided.

\section{SCATTER-POINT RESPONSE AND THE PSTM VELOCITY MODEL}

Here, we derive the $C$-wave diffraction curve from a point scatter in VTI media. Considering a scatter point at $(y, z)$ located immediately below a stack of VTI layers (Figure 4), we have (see Appendix A),

$$
\begin{aligned}
& t_{C}= \sqrt{\left(\frac{t_{C 0}}{1+\gamma_{0}}\right)^{2}+\frac{(y+h)^{2}}{V_{P 2}^{2}}-2 \eta_{e f f} \Delta t_{P}^{2}}+ \\
& \sqrt{\left(\frac{\gamma_{0} t_{C 0}}{1+\gamma_{0}}\right)^{2}+\frac{(y-h)^{2}}{V_{S 2}^{2}}+2 \zeta_{e f f} \Delta t_{S}^{2}}
\end{aligned}
$$

where

$$
\begin{aligned}
\Delta t_{P}^{2} & =\frac{(y+h)^{4}}{V_{P 2}^{2}\left[t_{C 0}^{2} V_{P 2}^{2} /\left(1+\gamma_{0}\right)^{2}+\left(1+2 \eta_{\text {eff }}\right)(y+h)^{2}\right]}, \text { and } \\
\Delta t_{S}^{2} & =\frac{(y-h)^{4}}{V_{S 2}^{2}\left[t_{C 0}^{2} V_{S 2}^{2} \gamma_{0}^{2} /\left(1+\gamma_{0}\right)^{2}+(y-h)^{2}\right]},
\end{aligned}
$$

where $h$ is the half source-receiver offset, and $t_{\mathrm{C} 0}$ is the vertical two-way C-wave time from the scatter point to the surface.

Equation (7) is accurate for offset-depth ratio of 2.5 (Yuan 2001; Li et al 2003). It contains five parameters: $\gamma_{0}, V_{\mathrm{P} 2}, V_{\mathrm{S} 2}, \eta_{\mathrm{eff}}$ and $\zeta_{\text {eff }}$, controlling the process of $C$-wave prestack time migration (PSTM). Of which, the four parameters $V_{\mathrm{P} 2}, V_{\mathrm{S} 2}, \eta_{\mathrm{eff}}$ and $\zeta_{\text {eff }}$ are referred to as the $C$-wave PSTM velocity model. With these formulations, the essential issue in processing converted-wave data is how to estimate these parameters, that is, model building.

\section{Links to the stacking velocity model}




\section{Li et al.}

A key question for model building is how to construct the PSTM velocity model from the stacking velocity model, since the stacking velocity model is often determined first during seismic data processing. Based on the definition in equation (3), we have,

$$
V_{P 2}^{2}=V_{C 2}^{2} \frac{\gamma_{e f f}\left(1+\gamma_{0}\right)}{1+\gamma_{e f f}}, V_{S 2}^{2}=V_{C 2}^{2} \frac{\left(1+\gamma_{0}\right)}{\gamma_{0}\left(1+\gamma_{e f f}\right)} .
$$

This defines the link between $\left(V_{\mathrm{P} 2}, V_{\mathrm{S} 2}\right)$ and $\left(V_{\mathrm{C} 2}, \gamma_{0}, \gamma_{\mathrm{eff}}\right)$.

The link between the anisotropic parameters is less obvious. Strictly speaking, a laystripping procedure based on equation (5) is required to obtain $\eta$ and $\zeta$ from $\chi$, as discussed in Dai and Li (2001). This may be necessary for depth processing, but for time processing simple empirical relationships based on the single-layer case may work better as found by Alkhalifah and Tsvankin (1995). According to equation (6), an empirical relationship $\zeta_{\text {eff }}=\eta_{\text {eff }} \gamma_{\text {eff }}^{2}$ (strictly true for a single layer) may be adopted to calculate $\zeta$ from $\eta$. Substituting this empirical relationship ( $\zeta_{\text {eff }}=\eta_{\text {eff }} \gamma_{\text {eff }}^{2}$ ) into equation (4), gives,

$$
\eta_{e f f}=\frac{\chi_{\text {eff }}}{\left(\gamma_{0}-1\right) \gamma_{\text {eff }}^{2}} ; \zeta_{\text {eff }}=\frac{\chi_{\text {eff }}}{\left(\gamma_{0}-1\right)} \text {. }
$$

We use numerical analysis to evaluate the accuracy of equation (10) and its effects on traveltime calculation. A five-layer model is created for this purpose, and the model parameters are shown in Table 1 . First, exact $\eta_{\text {eff }}$ and $\zeta_{\text {eff }}$ are calculated using equation (5) for the model; then exact $\chi_{\text {eff }}$ is calculated using equation (4); finally; we use equation (10) to recalculate the approximate $\eta_{\text {eff }}$ and $\zeta_{\text {eff. }}$ Figure 5 compares the exact ones with the approximate ones. As shown in Figures $5 \mathrm{a}$ and $5 \mathrm{~b}$, the differences are small in the shallow part (less than $2.0 \mathrm{~s}$ ); the error increases slightly beyond $2.0 \mathrm{~s}$, but is still within the error margin, since the resolution of velocity analysis usually decreases with depth. Furthermore, the effects of these

differences in $\eta_{\text {eff }}$ and $\zeta_{\text {eff }}$ on the calculation of traveltime are also negligible (Li and Yuan 2003). Moreover, the traveltime difference can be minimized by choosing another value of $\chi_{\text {eff. }}$ Therefore, for time processing, there exist a set of $\chi_{\text {eff }}$ that fits the traveltime. In this sense, equation (10) is a very good empirical relationship between the anisotropic parameters.

To sum up, for time processing, there is one-to-one analytical relationship between the stacking velocity model and the PSTM velocity model. This bridges the gap in model building.

\section{The diffraction curve in terms of the moveout attributes}

Substitute equations (9) and (10) into (7) and (8), we obtain expressions of the point diffraction in terms of the stacking velocity model $\left(V_{\mathrm{C} 2}, \gamma_{0}, \gamma_{\text {eff }}\right.$ and $\left.\chi_{\text {eff }}\right)$ : 


$$
\begin{aligned}
t_{C}= & \frac{1}{1+\gamma_{0}} \sqrt{t_{C 0}^{2}+\frac{\left(1+\gamma_{0}\right)\left(1+\gamma_{e f f}\right)}{\gamma_{e f f}} \frac{(y+h)^{2}}{V_{C 2}^{2}}-\frac{\left(1+\gamma_{0}\right)^{2}}{\left(\gamma_{0}-1\right) \gamma_{e f f}^{2}} 2 \chi_{e f f} \Delta t_{P}^{2}}+ \\
& \frac{\gamma_{0}}{1+\gamma_{0}} \sqrt{t_{C 0}^{2}+\frac{\left(1+\gamma_{0}\right)\left(1+\gamma_{e f f}\right)}{\gamma_{0}} \frac{(y-h)^{2}}{V_{C 2}^{2}}-\frac{\left(1+\gamma_{0}\right)^{2}}{\left(\gamma_{0}-1\right) \gamma_{0}^{2}} 2 \chi_{e f f} \Delta t_{S}^{2}}
\end{aligned},
$$

where

$$
\begin{aligned}
& \Delta t_{P}^{2}=\frac{\left(1+\gamma_{e f f}\right)(y+h)^{4}}{\gamma_{e f f} V_{C 2}^{2}\left[t_{C 0}^{2} V_{C 2}^{2} \gamma_{e f f} /\left(1+\gamma_{e f f}\right)+\left(1+\gamma_{0}\right)\left(1+\frac{2 \chi_{e f f}}{\left(\gamma_{0}-1\right) \gamma_{e f f}^{2}}\right)(y+h)^{2}\right]}, \text { and } \\
& \Delta t_{S}^{2}=\frac{\gamma_{0}\left(1+\gamma_{e f f}\right)(y-h)^{4}}{V_{C 2}^{2}\left[t_{C 0}^{2} V_{C 2}^{2} \gamma_{0} /\left(1+\gamma_{e f f}\right)+\left(1+\gamma_{0}\right)(y-h)^{2}\right]}
\end{aligned}
$$

We may directly use equation (11) to perform Kirckhoff PSTM based on the stacking velocity model.

\section{Processing strategy}

Therefore, it is now possible to use the following strategy to process converted-waves in anisotropic media: 1) using the zero-dip response [Equations (1) and (2)] to perform anisotropic velocity analysis to obtain the stacking velocity model $\left(V_{\mathrm{C} 2}, \gamma_{0}, \gamma_{\text {eff }}\right.$ and $\left.\chi_{\text {eff }}\right)$ in the ACP domain; 2) the obtained ( $V_{\mathrm{C} 2}, \gamma_{0}, \gamma_{\text {eff }}$ and $\left.\chi_{\text {eff }}\right)$ are then input to equation (11) to perform PSTM (the first pass) to build CIP gathers for migration velocity analysis; 3 ) using the CIP gathers to update the migration velocity model, which will be discussed in the next section; 4) after that, a second pass of PSTM is performed on the data to obtain the final imaging. In this way, we have successfully replaced CCP binning, DMO and post stack migration by converted-wave PSTM.

\section{MIGRATION VELOCITY ANALYSIS AND MODEL UPDATING}

Velocity model updating is necessary for two reasons: first the stacking velocity model $\left(V_{\mathrm{C} 2}\right.$, $\gamma_{\text {eff }}$ and $\left.\chi_{\text {eff }}\right)$ is defined at the ACP positions, and secondly the migration velocity may differ from the stacking velocity in layered media. The purpose of updating is to find the correct velocity model at the CIP positions that flattens the events in the CIP gathers. Here, we extend 
the concept of $\mathrm{P}$-wave migration velocity analysis to $\mathrm{C}$-wave and present a quantitative procedure to update the $\mathrm{C}$-wave migration velocity $V_{\text {Cmig. }}$. In principle, updating is achieved by analyzing the residual moveout in CIP gathers.

\section{C-wave migration velocity $\mathbf{V}_{\text {Cmig }}$}

Harrison (1992) studied the C-wave migration velocity for isotropic media, but his expression for $V_{\text {Cmig }}$ is of a complicated form. Here, we re-derive the migration velocity based on the diffraction curve defined in equation (7) in order to obtain simpler expressions that are valid for both layered isotropic and VTI media.

The migration velocity $V_{\text {Cmig }}$ is defined from the zero-offset diffraction curve $\left[t_{\mathrm{C}}(h=0)\right.$, equation (7)] as in the case of post-stack migration. For prestack migration, we can define the migration velocity from the midpoint diffraction curve $\left[t_{\mathrm{C}}(y=0)\right.$, equation (7)]. Since the two curves, $t_{\mathrm{C}}(h=0)$ and $t_{\mathrm{C}}(y=0)$, have exactly the same form, the two migration velocities are thus the same. As shown in Appendix B, similar to the moveout signature, the midpoint diffraction curve can also be decomposed into a hyperbolic term at near offsets and a non-hyperbolic term at far offsets, and the C-wave migration velocity $V_{\text {Cmig }}$ has the following relationship with the $\mathrm{P}$ - and S-wave stacking velocities:

$$
\frac{1}{V_{\text {Cmig }}^{2}}=\frac{1}{4}\left(\frac{1+\gamma_{0}}{V_{P 2}^{2}}+\frac{1+\gamma_{0}}{\gamma_{0} V_{S 2}^{2}}\right) .
$$

Substituting equation (9) into (13) gives,

$$
\frac{V_{C 2}}{V_{\text {Cmig }}}=\frac{1+\gamma_{\text {eff }}}{2 \sqrt{\gamma_{\text {eff }}}}
$$

These equations are valid for both isotropic and VTI media and they reduce to Harrison's (1992) equation in the isotropic single-layer case. Equation (13) reveals that generally speaking, the $\mathrm{C}$-wave stacking velocity $V_{\mathrm{C} 2}$ does not equal to the $\mathrm{C}$-wave migration velocity $V_{\text {Cmig }}$, and their difference is determined by the effective velocity ratio $\gamma_{\text {eff. }}$.

Substituting equation (14) into equations (11), and making some manipulations, gives,

$$
\begin{aligned}
t_{C}= & \frac{1}{1+\gamma_{0}} \sqrt{t_{C 0}^{2}+\frac{\left(1+\gamma_{0}\right)}{\left(1+\gamma_{e f f}\right)} \frac{(2 y+2 h)^{2}}{V_{C m i g}^{2}}-\frac{\left(1+\gamma_{0}\right)^{2}}{\left(\gamma_{0}-1\right) \gamma_{e f f}^{2}} 2 \chi_{e f f} \Delta t_{P}^{2}}+ \\
& \frac{\gamma_{0}}{1+\gamma_{0}} \sqrt{t_{C 0}^{2}+\frac{\left(1+\gamma_{0}\right) \gamma_{e f f}}{\left(1+\gamma_{e f f}\right) \gamma_{0}} \frac{(2 y-2 h)^{2}}{V_{C m i g}^{2}}-\frac{\left(1+\gamma_{0}\right)^{2}}{\left(\gamma_{0}-1\right) \gamma_{0}^{2}} 2 \chi_{e f f} \Delta t_{S}^{2}}
\end{aligned} .
$$

where 


$$
\begin{aligned}
& \Delta t_{P}^{2}=\frac{(2 y+2 h)^{4}}{\left(1+\gamma_{\text {eff }}\right) V_{C m i g}^{2}\left[t_{C 0}^{2} V_{C m i g}^{2}\left(1+\gamma_{\text {eff }}\right)+\left(1+\gamma_{0}\right)\left(1+\frac{2 \chi_{\text {eff }}}{\left(\gamma_{0}-1\right) \gamma_{\text {eff }}^{2}}\right)(2 y+2 h)^{2}\right]}, \text { and } \\
& \Delta t_{S}^{2}=\frac{\gamma_{\text {eff }}^{2} \gamma_{0}(2 y-2 h)^{4}}{\left(1+\gamma_{\text {eff }}\right) V_{\text {Cmig }}^{2}\left[t_{C 0}^{2} V_{\text {Cmig }}^{2} \gamma_{0}\left(1+\gamma_{\text {eff }}\right)+\left(1+\gamma_{0}\right) \gamma_{\text {eff }}(2 y-2 h)^{2}\right]} .
\end{aligned}
$$

Equation (15) can be used to perform the final migration once the migration velocity model is determined. Further model updating can also be performed using equation (15) if required.

Based on the above approach, migration velocity analysis requires the construction of common-midpoint (CMP) diffraction gathers, since the migration velocity is defined from the midpoint diffraction curve.

\section{Constructing CMP diffraction gathers}

The migration process has two functions. The first function is to migrate data spatially for correctly positioning the events, and the second function is to time-shift the data to correct for the moveout, correctly focusing the energy by stacking the flattened events. The migration output can be either a migrated image section, or migrated CIP gathers, as intermediate results. If the correct velocity model is used for migration, all events in the CIP gather should be flat, properly positioned; stacking the CIP gathers focuses the energy and yields the migrated section. Then, one may wonder what effect it will have on the data if the velocity model is incorrect?

A commonly used assumption in migration velocity analysis is that the positioning process during migration is weakly dependent on the velocity model, whilst the time-shifting process is strongly dependent on the velocity model. This means that the function of positioning an event in a CIP gather will still largely be fulfilled even if a slightly-deviated velocity model is used, but the moveout may not be properly corrected, inducing residual moveouts. These residual moveouts may then be used to update the model. Here we use the same assumptions for $\mathrm{C}$-wave migration velocity analysis.

For near-offset data, the time-shifting process due to migration is equivalent to applying a hyperbolic NMO correction to the data controlled by $V_{\mathrm{Cmig}}$, because the C-wave diffraction curve contains a hyperbolic term for near-offsets (See below and Appendix C). Therefore, after migration, these time-shifts can, in theory, be removed by apply an inverse NMO to the CIP gather, as long as the positioning of the events are judged to be satisfactory. In this way, we can obtain adequately positioned CIP gather but with the original moveout retained. This 


\section{Li et al.}

gather simulates the CMP diffraction gather if the midpoint diffraction curve is used to remove the time-shifts due to migration.

In Appendices B and C, we have derived the Taylor series expansion of the midpoint diffraction curve,

$$
t^{2}=t_{C 0}^{2}+\frac{x^{2}}{V_{C m i g}^{2}}+\frac{D_{4} x^{4}}{1+D_{5} x^{2}}
$$

where

$$
\begin{aligned}
& D_{4}=-\frac{\left(\gamma_{0}-\gamma_{e f f}\right)^{2}+8\left(1+\gamma_{0}\right)\left(\gamma_{0} \eta_{e f f}-\gamma_{e f f}^{2} \zeta_{e f f}\right)}{4 t_{C 0}^{2} V_{C m i g}^{4} \gamma_{0}\left(1+\gamma_{e f f}\right)^{2}}, \text { and } \\
& D_{5}=\frac{D_{4}}{\frac{1}{4}\left(\frac{1}{V_{P h}}+\frac{1}{V_{S h}}\right)^{2}-\frac{1}{V_{C m i g}^{2}}} .
\end{aligned}
$$

and $x=2 h$ is the source and receiver offset (Figure 4). Equations (17) and (18) can then be used to perform migration velocity analysis for updating $V_{\text {Cmig }}$ from a CMP diffraction gather (CIP gathers with the original moveout retained).

The construction of the CMP diffraction gather is dependent on the quality of positioning which is weakly dependent on the velocity model. The positioning of the events in the CMP diffraction gathers improves gradually as the model converges to the correct model. Dai and Li (2005) showed that this process converges to the correct velocity after only one or two iterations.

\section{Work flow for migration velocity analysis}

The following work flow can be used to perform migration velocity analysis: 1) after obtaining the stacking velocity model, equation (11) is used to create CIP gathers; 2) an inversionmoveout correction is then applied to the CIP gathers based on the midpoint diffraction curve $\left[t_{\mathrm{C}}(y=0)\right]$, yielding the CMP diffraction gathers, 3) apply equation (17) and (18) to re-estimate the migration velocity from these CMP diffraction gathers; 4) using equation (15) for final PSTM, or further model updating if needed.

\section{D DATA EXAMPLE}

The above PSTM scheme wasapplied to several 4C datasets from the North Sea, including Valhall, Manhogany (Li et al. 2001); Alba (Dai and Li 2001). Here we select two recent examples for illustrative purposes. A 2D example is presented in this section and 3D example 
in the next section. The results of the new scheme are compared with the conventional approach of DMO-poststack migration.

The first example is a 2D 4C data from Lomond, North Sea (courtesy of BG plc), where 4C data are used to improve imaging beneath gas clouds. More details of this dataset can be found in Mancini et al, 2002. In the study area, the reservoir cannot be imaged properly using $\mathrm{P}$-wave due to the presence of gas cloud. Figure 6a shows a conventional P-wave section acquired in Lomond. In the target zone, there is a pull-down effect due to the gas cloud and the imaging is blurred. The $4 \mathrm{C}$ data set was then acquired in order to undershoot the gas cloud.

\section{Estimating $\gamma_{0}$}

Figure 6 illustrates the correlation analysis for determining $\gamma_{0}$, where we have successfully correlated five events between the $P$ - and $C$-wave sections. Correlation is based on structural similarity through visual inspection. As one can see, a detailed correlation is not necessary. Li and Yuan (2003) shows that time processing allows $10-15 \%$ error margins in $\gamma_{0}$.

\section{Estimating $\mathbf{V}_{C 2}, \gamma_{e f f}$ and $\chi_{e f f}$.}

For practical applications, an interactive tool is developed for estimating the three moveout parameters (Dai 2003). Figure 7 illustrates this tool for determining $V_{\mathrm{C} 2}, \gamma_{\text {eff }}$ and $\chi_{\text {eff. }}$ The farright panel illustrates the input common ACP gather. The far-left panel displays the $V_{\mathrm{C} 2}$ spectra for interactive picking. The second panel from the left displays the velocity ratios, the red curve shows the input $\gamma_{0}$, and the other curve shows $\gamma_{\text {eff. }}$ The third panel displays the anisotropic coefficient $\chi_{\text {eff. }}$. The flatness of an event over the intermediate and far offset ranges determines the values of $\gamma_{\text {eff }}$ and $\chi_{\text {eff }}$ through visual inspection.

\section{Model updating}

A similar interactive tool as in Figure 7 is used to perform model updating. In this case, the input is a CMP diffraction gather instead of a common ACP gather. Figure 8 illustrates this process. First, interactive velocity analysis is performed over common ACP gathers to determine the stacking velocity model $V_{\mathrm{C} 2}, \gamma_{\text {eff }}$ and $\chi_{\text {eff }}$ (Figure $8 \mathrm{a}$ ). Note that $\gamma_{0}$ is determined before this step by correlation analysis as in Figure $6 . V_{\text {Cmig }}$ is then calculated from the stacking model and a CIP gather is generated using equation (15), as shown Figure 8b. Since the events are not flat, an inverse NMO with $V_{\text {Cmig }}$ is applied to the CIP gather in Figure 8b, creating a CMP diffraction gather (Figure 8c). This gather is input to the interactive tool again for 


\section{Li et al.}

updating $V_{\mathrm{C} 2}$ and $\chi_{\text {eff }}$, and the resulted CIP gather then becomes flat (Figure $8 \mathrm{~d}$ ). This requires two NMO runs and two PSTM runs. Note that there is still some residual moveout in the final CIP gather in Figure 8d, and a post-migration residual moveout correction may be applied to improve the alignment further.

\section{Results and comparison}

The final PSTM results of this data are shown in Figure 9, compared with the DMO and poststack migrated results. There is a substantial improvement to the reservoir structure. But towards the shallow part of the section, the DMO results appear to be better than the PSTM imaging. This is due the fact that the near-offset field in the shallow section has better signal than the wavefield in the intermediate offsets, and a larger mute for the shallow events shall improve the results, as demonstrated in Mancini (2004). More significantly, the improvement over the target interval agrees with the well data as shown in Figure 10.

\section{D DATA EXAMPLE}

The second example is a 3D 4C dataset from the North Sea (Courtesy of Kerr-McGee North Sea Ltd.). The data was acquired in August 2001 with a nominal receiver area of $10.8 \mathrm{~km}^{2}$. It was acquired with an inline geometry using 2 cables and 2 swaths of shot lines. Each swath has 22 shot lines. The survey was centred on a domed, crestal structure which is obscured by a gas chimney. The purpose of acquiring $\mathrm{C}$-wave data is to define the top of the structure at the reservoir target level (3.0 seconds PP time and 5.5 seconds PS time). It is expected that the converted-wave processing will clarify structural details.

\section{Estimating the stacking velocity model}

For 3D data applications, $\gamma_{0}$ is estimated using an interactive correlation tool, as shown in Figure 11 . This is necessary for dealing with the large volumes of 3D data. The estimated $\gamma_{0}$ field is then calibrated in the borehole position based on well-ties. Once $\gamma_{0}$ is determined, $V_{\mathrm{C} 2}$, $\gamma_{\text {eff }}$ and $\chi_{\text {eff }}$ can be determined from common ACP gathers in the same way as in the $2 \mathrm{D}$ case. Figure 12 shows an example of this process.

\section{Model updating}

Similar to the 2D case, model updating is achieved through the analysis of CIP gathers. For $3 \mathrm{D}$, this is not a easy task, in particularly if the $3 \mathrm{D}$ velocity model varies with azimuth. In this 
dataset, we find that the azimuthal velocity variation is weak satisfying the condition of transverse isotropy (Dai et al, 2004). Therefore, the tools developed for the $2 \mathrm{D}$ case can be directly applied to this 3D dataset. Figure 13 shows an example of the velocity modelupdating and the corresponding CMP diffraction gather at CIP line 050 and CIP number 350 from the data of shot line 5002 and receiver cable line 1 in which the shot line and receiver line are at the same location.

\section{D PSTM results}

The final image at one CIP line is the summation of the relevant contributions from all shot and receiver line data. Figure 14 shows the migrated PP and C-wave images at the CIP line located in the middle of the two receiver cables, and both are displayed in PP time. The distance between the two cables is $500 \mathrm{~m}$. The target is at around 3 seconds of PP-time between CIP 350 and 650 and cannot be seen from the P-wave image (Figure 14a) due to the gas chimney. However, it can be seen clearly on the C-wave image, showing the structure of interest with crestal faulting under the gas chimney, and the faults in the target can also be clearly identified (Figure 14b).

\section{Drilling results and comparison}

Drilling results confirm the structure in the 3D migrated images. Figure 15 shows one example of the comparison between the images obtained by the PSTM approach and by the conventional DMO approach. Wells and interpreted horizons are marked on both images. The PSTM result clearly shows the structure with crestal faulting which is absent in the DMO result. Instead the DMO result reveals a possible anticline. Drilling results confirmed the structure imaged by PSTM (Paul Conway - personal communication). Note that the resolution in the PSTM result appears to be lower than the DMO result. This is mainly due to the difference in the offset ranges used to create the image. The DMO processing uses only nearoffset data with offset-depth rato up to $1.0(x / z<1.0)$, whilst the PSTM result utilizes data from all offsets, particularly, the data from the intermediate to far offsets ( $x / z$ from 1.5 to 2.0 )

\section{DISCUSSION AND CONCLUSIONS}




\section{Li et al.}

We have presented some basic theories for describing the C-wave moveout in layered VTI media which controls the stacking process, and for describing the diffraction from a point scatter which controls the process of prestack time migration. Both theory and application show that $C$-wave time processing in VTI media is determined by four parameters: $V_{\mathrm{C} 2}, \gamma_{0}, \gamma_{\mathrm{eff}}$ and $\chi_{\text {eff. }}$ Except $\gamma_{0}$, the other three parameters can be determined from the moveout signatures either in the ACP or in the CIP domain. This is because of each of these parameter controls a particular data aperture of primary influence: $V_{\mathrm{C} 2}$ for the near offsets $(x / z<1.0)$; $\gamma_{\text {eff }}$ for intermediate offsets $(x / z<1.5) ; \chi_{\text {eff }}$ for the far offsets $(x / z<2.0)$.

We have also derived a simplified relationship between the $\mathrm{C}$-wave migration velocity $V_{\mathrm{Cmig}}$ and stacking velocity $V_{\mathrm{C} 2}$, as well as their relationships with $\mathrm{P}$ - and S-wave stacking velocitites. Based on the midpoint diffraction curve $\left[t_{\mathrm{C}}(y=0)\right.$, equation (7)], we have introduced the concept of common midpoint (CMP) diffraction gathers. The midpoint diffraction curve at the near-offsets is close to a hyperbola controlled by $V_{\text {Cmig. }}$ Thus, we can construct CMP diffraction gathers through inverse NMO applied to the CIP gathers. These CMP diffraction gathers can then be used to update the migration velocity, and analytical expressions have also been derived for this purpose.

The construction of the CMP diffraction gathers relies on the separation of the migration process into a positioning process and a time-shifting (focusing) process, and the fact that the positioning process is less dependent on velocity than the time-shifting process. Applying inverse NMO to the CIP gathers can therefore reverse the time-shifting process. An alternative way to construct the CMP diffraction gather is by common-offset partial migration which is likely to be more robust and much less dependent on the velocity model.

The above leads to the development of a new processing scheme based on C-wave PSTM which replaces the sequence of CCP binning, DMO and poststack migration. Real data examples show that the new scheme improves the $\mathrm{C}$-wave imaging substantially, and that $C$ wave anisotropic imaging can be carried out accurately and low cost.

\section{ACKNOWLEDGEMENTS}

We thank BG plc for permission to show the Lomond 4C data, and Kerr-McGee North Sea (UK) Ltd, Talisman Energy, Total E\&P UK Ltd and ExxonMobil for permission to show the 3D 4C North Sea data. We also thank Tim Pointer of BG, and Paul Conway of KerrMcGee for various discussions related to the relevant data. This work is funded by the Edinburgh Anisotropy Project (EAP) of the British Geological Survey, and is published with the approval of the Executive Director of British Geological Survey (NERC) and the EAP sponsors: Agip, 
BG, BGP-CNPC, BP, Chevron, ConocoPhillips, ExxonMobil, GX Technology, Kerr-McGee, Landmark, Marathon, Norsk Hydro, PDVSA, Shell, Schlumberger, SinoPec, Total, Veritas DGC.

\section{REFERENCES}

Alkhalifah T. and Tsvankin I. 1995. Velocity analysis for transversely isotropic media. Geophysics 60, 1550-1566.

Audebert F., Granger P. Y. and Herrenschmidt A. 1999. CCP-scan technique: True common conversion point sorting and converted-wave velocity analysis solved by PP - PS prestack depth migration. 69th SEG Meeting, Houston, USA, Expanded Abstracts, 1186-1189.

Cheret T., Bale R. and Leaney S. 2000. Parameterization of polar anisotropic moveout for converted waves. $70^{\text {th }}$ SEG Meeting, Calgary, Canada, Expanded Abstracts, 11811184.

Dai H. 2003. Integrative analysis of anisotropy parameter and velocities for PS converted waves. 73rd SEG meeting, Dallas, USA, Expanded Abstracts, 1577-1580.

Dai H and Li X.-Y. 2005. Migration velocity analysis for PS converted waves: Part I: theory. Geophysical Prospecting, submitted.

Dai H. and Li X.-Y. 2001. Anisotropic migration and model building for 4-C seismic data: A case study from Alba. 71st SEG Meeting, San Antonio, USA, Expanded Abstracts, 795-798.

Dai H., Li X.-Y. and Conway P. 2004. 3D pre-stack Kirchhoff time migration of PS-waves and migration velocity model building, 74th SEG Meeting, Denver, USA, Expanded Abstracts, 1115-1118.

Hale I.D. 1984. 1984, Dip-moveout by Fourier transform. Geophysics 49, 741-757.

Harrison M.P. 1992. Processing of P-SV surface seismic data: anisotropy analysis, dip moveout and migration. Ph.D. thesis, The University of Calgary.

Li X.-Y. 2003. Converted-wave moveout analysis revisited: the search for a standard approach. 73rd SEG Meeting, Dallas, USA, Expanded Abstracts, 805-808.

Li X.-Y. and Druzhinin A. 2000. A practical approach to P-SV prestack time migration and velocity analysis for transverse isotropy. 70th SEG Meeting, Calgary, Canada, Expanded Abstracts, 1142-1145. 
18 Li et al.

Li X.-Y. and Yuan J. 2003. Converted-wave moveout and conversion-point equation in layered VTI media: theory and application. Journal Applied Geophysics 54, 297318.

Li X.-Y., Yuan J. and Bale R. 2003. Converted-wave traveltime equations in layered anisotropic media: An overview. $65^{\text {th }}$ EAGE Conference, Expanded Abstracts, P105.

Mancini F., Li. X.-Y., Ziolkowki A. and Pointer T. 2002. Interpreting velocity ratios from 4C seismic data and well logs in the presence of gas and anisotropy. 72nd SEG Meeting, Salt Lake City, USA, Expanded Abstract, 1002-1005.

Mancini F. 2004. Converted-wave imaging in anisotropic media using sea-floor seismic data. $\mathrm{PhD}$ thesis, University of Edinburgh.

Rommel B. E. 1996. Dip move out processing (DMO) for converted waves in transversely isotropic media. 58th EAGE Meeting, P136.

Stewart R.R., Gaiser J.E., Brown R.J. and Lawton D.C. 2002. Converted-wave seismic exploration: Methods. Geophysics 67, 1345-1363.

Tessmer G. and Behle A. 1988. Common-conversion point stacking technique for converted waves. Geophysical Prospecting 36, 671-688.

Thomsen L. 1986. Weak elastic anisotropy. Geophysics 51, 1954-1966.

Thomsen L. 1999. Converted-wave reflection seismology over inhomogeneous, anisotropic media. Geophysics 64, 678-690.

Tsvankin I. and Grechka V. 2000. Dip moveout of converted waves and parameter estimation in transversely isotropic media. Geophysical Prospecting 48, 257-292.

Tsvankin I. and Thomsen L. 1994. Nonhyperbolic reflection moveout in anisotropic media. Geophysics 59, 1290-1304.

Yuan J. 2001. Analysis of four-component seafloor seismic data for seismic anisotropy. Ph.D. thesis, The University of Edinburgh.

Zhang Y. 1996. Non-hyperbolic converted wave velocity analysis and normal moveout. $66^{\text {th }}$ SEG Meeting, Denver, USA, Expanded Abstracts, 1555-1558.

\section{APPENDIX A \\ DERIVATION OF THE C-WAVE DIFFRACTION EQUATION (7)}

From Figure 4, assuming the traveltime for the P-wave leg as $t_{\mathrm{P}}$, and for the S-wave leg as $t_{\mathrm{S}}$, the $\mathrm{C}$-wave diffraction curve can then be written as,

$$
t_{C}=t_{P}+t_{S} .
$$


From Alkhalifah and Tsvankin (1995), we have

$$
t_{P}=\sqrt{t_{P 0}^{2}+\frac{(y+h)^{2}}{V_{P 2}^{2}}-2 \eta_{e f f} \Delta t_{P}^{2}}
$$

where $t_{P 0}$ is the vertical one-wave P-wave time from the scatter point to the surface, and

$$
\Delta t_{P}^{2}=\frac{(y+h)^{4}}{V_{P 2}^{2}\left[t_{P 0}^{2} V_{P 2}^{2}+\left(1+2 \eta_{e f f}\right)(y+h)^{2}\right]} .
$$

Similarly, following Yuan (2001), we have,

$$
t_{S}=\sqrt{t_{S 0}^{2}+\frac{(h-y)^{2}}{V_{S 2}^{2}}-2 \zeta_{e f f} \Delta t_{S}^{2}},
$$

where $t_{S 0}$ is the vertical one-wave S-wave time from the scatter point to the surface, and

$$
\Delta t_{S}^{2}=\frac{(y-h)^{4}}{V_{S 2}^{2}\left[t_{S 0}^{2} V_{S 2}^{2}+(y-h)^{2}\right]} .
$$

Substituting (A-2) and (A-4 into (A-1), we have,

$$
t_{C}=\sqrt{t_{P 0}^{2}+\frac{(y+h)^{2}}{V_{P 2}^{2}}-2 \eta_{\text {eff }} \Delta t_{P}^{2}}+\sqrt{t_{S 0}^{2}+\frac{(y-h)^{2}}{V_{S 2}^{2}}-2 \zeta_{\text {eff }} \Delta t_{S}^{2}},
$$

Note that,

$$
t_{P 0}=\frac{1}{1+\gamma_{0}} t_{C 0} ; t_{S 0}=\frac{\gamma_{0}}{1+\gamma_{0}} t_{C 0}
$$

where $t_{\mathrm{C} 0}\left(=t_{\mathrm{P} 0}+t_{\mathrm{S} 0}\right)$ is the vertical two-way $\mathrm{C}$-wave time from the scatter point to the surface. Substituting equations (A-7), into equation (A-6) gives equation (7).

\section{APPENDIX B \\ DERIVING THE C-WAVE MIGRATION VELOCITY $V_{\text {CMIG }}$}

For poststack migration, the migration velocity is defined by the zero-offset diffraction curve, which can be obtained from the diffraction equation by letting $h=0$. Here for prestack 
migration, we define the migration velocity from the midpoint diffraction curve by letting $y=0$. These two curves have the same form and the two velocities are equal. Denoting the midpoint diffraction time as $t$ for simplicity, and letting $y=0$ in equation (A-6), gives,

$$
t=t_{C}(y=0)=\sqrt{t_{P 0}^{2}+\frac{h^{2}}{V_{P 2}^{2}}-2 \eta_{e f f} \Delta t_{P}^{2}(y=0)}+\sqrt{t_{S 0}^{2}+\frac{h^{2}}{V_{S 2}^{2}}-2 \zeta_{\text {eff }} \Delta t_{S}^{2}(y=0)},
$$

where,

$$
\begin{aligned}
& \Delta t_{P}^{2}(y=0)=\frac{h^{4}}{V_{P 2}^{2}\left[t_{P 0}^{2} V_{P 2}^{2}+\left(1+2 \eta_{\text {eff }}\right) h^{2}\right]} \\
& \Delta t_{S}^{2}(y=0)=\frac{h^{4}}{V_{S 2}^{2}\left[t_{S 0}^{2} V_{S 2}^{2}+h^{2}\right]}
\end{aligned}
$$

Squaring both sides of (B-1) gives,

$$
\begin{aligned}
& t^{2}=f\left(h^{2}\right)=t_{P}^{2}+t_{S}^{2}+2 t_{P} t_{S} \\
& t_{P}^{2}=t_{P 0}^{2}+\frac{h^{2}}{V_{P 2}^{2}}-2 \eta_{e f f} \Delta t_{P}^{2}, t_{P}=\sqrt{t_{P 0}^{2}+\frac{h^{2}}{V_{P 2}^{2}}-2 \eta_{e f f} \Delta t_{P}^{2}}, \\
& t_{S}^{2}=t_{S 0}^{2}+\frac{h^{2}}{V_{S 2}^{2}}+2 \zeta_{\text {eff }} \Delta t_{S}^{2}, t_{S}=\sqrt{t_{S 0}^{2}+\frac{h^{2}}{V_{S 2}^{2}}+2 \zeta_{\text {eff }} \Delta t_{S}^{2} .}
\end{aligned}
$$

Expanding the $t^{2}\left(h^{2}\right)$ curve in Taylor series near $h^{2}=0$ gives,

$$
t^{2}=D_{0}+D_{2} 2^{2} h^{2}+D_{4} 2^{4} h^{4}+\ldots
$$

where

$$
D_{0}=f(0)=t_{P 0}+t_{S 0}=t_{C 0}, D_{2}=\left.\frac{1}{4} \frac{d t^{2}}{d h^{2}}\right|_{h=0}, D_{4}=\left.\frac{1}{32} \frac{d}{d h^{2}}\left(\frac{d t^{2}}{d h^{2}}\right)\right|_{h=0}
$$

The migration velocity is defined as,

$$
\frac{1}{V_{\text {Cmig }}^{2}}=D_{2}
$$

From (B-1), we can obtain the derivatives as follows, 


$$
\begin{aligned}
& \frac{d t^{2}}{d h^{2}}=\frac{d t_{P}^{2}}{d h^{2}}+\frac{d t_{S}^{2}}{d h^{2}}+2 t_{S} \frac{d t_{P}}{d h^{2}}+2 t_{P} \frac{d t_{S}}{d h^{2}}=\left(1+\frac{t_{S}}{t_{P}}\right) \frac{d t_{P}^{2}}{d h^{2}}+\left(1+\frac{t_{P}}{t_{S}}\right) \frac{d t_{S}^{2}}{d h^{2}}, \\
& \frac{d}{d h^{2}}\left(\frac{d t^{2}}{d h^{2}}\right)=\frac{d}{d h^{2}}\left(\frac{d t_{P}^{2}}{d h^{2}}\right)+\frac{d}{d h^{2}}\left(\frac{d t_{S}^{2}}{d h^{2}}\right)+4 \frac{d t_{P}}{d h^{2}} \frac{d t_{S}}{d h^{2}}+2 t_{S} \frac{d}{d h^{2}}\left(\frac{d t_{P}}{d h^{2}}\right)+2 t_{P} \frac{d}{d h^{2}}\left(\frac{d t_{S}}{d h^{2}}\right)= \\
& =\left(1+\frac{t_{S}}{t_{P}}\right) \frac{d}{d h^{2}}\left(\frac{d t_{P}^{2}}{d h^{2}}\right)+\left(1+\frac{t_{P}}{t_{S}}\right) \frac{d}{d h^{2}}\left(\frac{d t_{S}^{2}}{d h^{2}}\right)+\frac{1}{t_{P} t_{S}} \frac{d t_{P}^{2}}{d h^{2}} \frac{d t_{S}^{2}}{d h^{2}}-\frac{t_{S}}{2 t_{P}^{3}}\left(\frac{d t_{P}^{2}}{d h^{2}}\right)^{2}-\frac{t_{P}}{2 t_{S}^{3}}\left(\frac{d t_{S}^{2}}{d h^{2}}\right)^{2}= \\
& =\left(1+\frac{t_{S}}{t_{P}}\right) \frac{d}{d h^{2}}\left(\frac{d t_{P}^{2}}{d h^{2}}\right)+\left(1+\frac{t_{P}}{t_{S}}\right) \frac{d}{d h^{2}}\left(\frac{d t_{S}^{2}}{d h^{2}}\right)-\frac{1}{2 t_{P} t_{S}}\left[\frac{t_{S}}{t_{P}}\left(\frac{d t_{P}^{2}}{d h^{2}}\right)-\frac{t_{P}}{t_{S}}\left(\frac{d t_{S}^{2}}{d h^{2}}\right)\right]^{2},
\end{aligned}
$$

noting that,

$$
\begin{aligned}
& \frac{d t_{P}}{d h^{2}}=\frac{1}{2 t_{P}} \frac{d t_{P}^{2}}{d h^{2}}, \frac{d t_{S}}{d h^{2}}=\frac{1}{2 t_{S}} \frac{d t_{S}^{2}}{d h^{2}}, \\
& \frac{d}{d h^{2}}\left(\frac{d t_{P}}{d h^{2}}\right)=\frac{1}{2 t_{P}} \frac{d}{d h^{2}}\left(\frac{d t_{P}^{2}}{d h^{2}}\right)-\frac{1}{4 t_{P}^{3}}\left(\frac{d t_{P}^{2}}{d h^{2}}\right)^{2}, \frac{d}{d h^{2}}\left(\frac{d t_{S}}{d h^{2}}\right)=\frac{1}{2 t_{S}} \frac{d}{d h^{2}}\left(\frac{d t_{S}^{2}}{d h^{2}}\right)-\frac{1}{4 t_{S}^{3}}\left(\frac{d t_{S}^{2}}{d h^{2}}\right)^{2} .
\end{aligned}
$$

Substituting (B-7) and (B-8) into (B-5), gives,

$$
\begin{gathered}
4 D_{2}=\left.\frac{d t^{2}}{d h^{2}}\right|_{h=0}=\left.\left(1+\gamma_{0}\right) \frac{d t_{P}^{2}}{d h^{2}}\right|_{h=0}+\left.\left(1+\frac{1}{\gamma_{0}}\right) \frac{d t_{S}^{2}}{d h^{2}}\right|_{h=0}, \\
32 D_{4}=\left.\frac{d}{d h^{2}}\left(\frac{d t^{2}}{d h^{2}}\right)\right|_{h=0}=\left.\left(1+\gamma_{0}\right) \frac{d}{d h^{2}}\left(\frac{d t_{P}^{2}}{d h^{2}}\right)\right|_{h=0}+\left.\left(1+\frac{1}{\gamma_{0}}\right) \frac{d}{d h^{2}}\left(\frac{d t_{S}^{2}}{d h^{2}}\right)\right|_{h=0}- \\
-\frac{1}{2 t_{P 0} t_{S 0}}\left[\left.\gamma_{0}\left(\frac{d t_{P}^{2}}{d h^{2}}\right)\right|_{h=0}-\left.\frac{1}{\gamma_{0}}\left(\frac{d t_{S}^{2}}{d h^{2}}\right)\right|_{h=0}\right]^{2} .
\end{gathered}
$$

Therefore, we need to calculate the first and second derivatives of function $t_{P}^{2}\left(h^{2}\right)$ and $t_{S}^{2}\left(h^{2}\right)$ against variable $h^{2}$, and evaluate their values at $h=0$, respectively. From equation (B3), we have,

$$
\begin{aligned}
& \left.\frac{d t_{P}^{2}}{d h^{2}}\right|_{h=0}=\frac{1}{V_{P 2}^{2}}-\left.2 \eta_{e f f} \frac{d \Delta t_{P}^{2}}{d h^{2}}\right|_{h=0},\left.\frac{d}{d h^{2}}\left(\frac{d t_{P}^{2}}{d h^{2}}\right)\right|_{h=0}=-\left.2 \eta_{\text {eff }} \frac{d}{d h^{2}}\left(\frac{d \Delta t_{P}^{2}}{d h^{2}}\right)\right|_{h=0} \\
& \left.\frac{d t_{S}^{2}}{d h^{2}}\right|_{h=0}=\frac{1}{V_{S 2}^{2}}+\left.2 \zeta_{\text {eff }} \frac{d \Delta t_{S}^{2}}{d h^{2}}\right|_{h=0},\left.\frac{d}{d h^{2}}\left(\frac{d t_{S}^{2}}{d h^{2}}\right)\right|_{h=0}=\left.2 \zeta_{e f f} \frac{d}{d h^{2}}\left(\frac{d \Delta t_{S}^{2}}{d h^{2}}\right)\right|_{h=0}
\end{aligned}
$$


From equation (B-2), we have,

$$
\begin{aligned}
& \left.\frac{d \Delta t_{P}^{2}}{d h^{2}}\right|_{h=0}=0,\left.\quad \frac{d}{d h^{2}}\left(\frac{d \Delta t_{P}^{2}}{d h^{2}}\right)\right|_{h=0}=\frac{2}{t_{P 0}^{2} V_{P 2}^{4}} \\
& \left.\frac{d \Delta t_{P}^{2}}{d h^{2}}\right|_{h=0}=0,\left.\quad \frac{d}{d h^{2}}\left(\frac{d \Delta t_{S}^{2}}{d h^{2}}\right)\right|_{h=0}=\frac{2}{t_{S 0}^{2} V_{S 2}^{4}}
\end{aligned}
$$

Substituting (B-12) into (B-11), gives

$$
\begin{aligned}
& \left.\frac{d t_{P}^{2}}{d h^{2}}\right|_{h=0}=\frac{1}{V_{P 2}^{2}},\left.\frac{d}{d h^{2}}\left(\frac{d t_{P}^{2}}{d h^{2}}\right)\right|_{h=0}=-\frac{4 \eta_{e f f}}{t_{P 0}^{2} V_{P 2}^{4}} \\
& \left.\frac{d t_{S}^{2}}{d h^{2}}\right|_{h=0}=\frac{1}{V_{S 2}^{2}},\left.\frac{d}{d h^{2}}\left(\frac{d t_{S}^{2}}{d h^{2}}\right)\right|_{h=0}=\frac{4 \zeta_{\text {eff }}}{t_{S 0}^{2} V_{S 2}^{4}}
\end{aligned}
$$

Substituting (B-13) into (B-9) and (B-10), gives

$$
\begin{aligned}
& 4 D_{2}=\left.\frac{d t^{2}}{d h^{2}}\right|_{h=0}=\frac{1+\gamma_{0}}{V_{P 2}^{2}}+\frac{1+\gamma_{0}}{\gamma_{0} V_{S 2}^{2}}, \\
& 32 D_{4}=\left.\frac{d}{d h^{2}}\left(\frac{d t^{2}}{d h^{2}}\right)\right|_{h=0}=\frac{-2\left(1+\gamma_{0}\right) \eta_{e f f}}{t_{P 0}^{2} V_{P 2}^{4}}+\frac{2\left(1+\gamma_{0}\right) \zeta_{e f f}}{\gamma_{0} t_{S 0}^{2} V_{S 2}^{4}}-\frac{1}{4 t_{P 0} t_{S 0}}\left(\frac{\gamma_{0}}{V_{P 2}^{2}}-\frac{1}{\gamma_{0} V_{S 2}^{2}}\right)^{2} .(\mathrm{B}-15)
\end{aligned}
$$

Substituting (B-14) into (B-6), gives,

$$
\frac{1}{V_{\text {Cmig }}^{2}}=\frac{1}{4}\left(\frac{1+\gamma_{0}}{V_{P 2}^{2}}+\frac{1+\gamma_{0}}{\gamma_{0} V_{S 2}^{2}}\right) \text {. }
$$

\section{APPENDIX C}

\section{THE TAYLOR SERIES EQUATION FOR THE CMP DIFFRACTION CURVE}

Similar to the moveout signature, we can also write the Taylor series expansion of the midpoint diffraction curve as,

$$
t^{2}=t_{C 0}^{2}+\frac{4 h^{2}}{V_{\text {Cmig }}^{2}}+\frac{16 D_{4} h^{4}}{1+4 D_{5} h^{2}},
$$

where $D_{5}$ controls the far-offset diffraction, and can be determined from the horizontal velocities of P-wave and S-waves. Note that, 


$$
\left.\frac{d t^{2}}{d h^{2}}\right|_{h=\infty}=\left(\frac{1}{V_{P h}}+\frac{1}{V_{S h}}\right)^{2}=\frac{1}{V_{h}^{2}}=\frac{4}{V_{C m i g}^{2}}+\frac{4 D_{4}}{D_{5}},
$$

where $V_{\mathrm{Ph}}$ and $V_{\mathrm{Sh}}$ are the $\mathrm{P}$ - and S-wave horizontal velocities, respectively $V_{\mathrm{h}}$ is the average horizontal velocities. Solving (C-2) for $D_{5}$ gives,

$$
D_{5}=\frac{D_{4}}{\frac{1}{4} \frac{1}{V_{h}^{2}}-\frac{1}{V_{C m i g}^{2}}} .
$$

Utilizing the relationships between $V_{\mathrm{P} 2}, V_{\mathrm{S} 2}$ and $V_{\mathrm{C} 2}$ [Equation (9)], and between $V_{\mathrm{C} 2}$ and $V_{\mathrm{Cmig}}$ [equation (14)], we can obtain,

$$
V_{P 2}^{2}=\frac{1}{4} V_{C m i g}^{2}\left(1+\gamma_{0}\right)\left(1+\gamma_{e f f}\right), \quad V_{S 2}^{2}=V_{C m i g}^{2} \frac{\left(1+\gamma_{0}\right)\left(1+\gamma_{e f f}\right)}{4 \gamma_{0} \gamma_{e f f}} .
$$

Substituting (C-4) into (B-15) gives a $D_{4}$ in terms of $V_{\mathrm{Cmig}}$,

$$
\begin{aligned}
D_{4} & =-\frac{\left(\gamma_{0}-\gamma_{e f f}\right)^{2}+8\left(1+\gamma_{0}\right)\left(\gamma_{0} \eta_{e f f}-\gamma_{e f f}^{2} \zeta_{e f f}\right)}{4 t_{C 0}^{2} V_{C m i g}^{4} \gamma_{0}\left(1+\gamma_{e f f}\right)^{2}}, \text { and } \\
D_{5} & =\frac{D_{4}}{\frac{1}{4}\left(\frac{1}{V_{P h}}+\frac{1}{V_{S h}}\right)^{2}-\frac{1}{V_{C m i g}^{2}}} .
\end{aligned}
$$

Equation (C-1) can be used to perform migration velocity analysis in CMP diffraction gathers. The current migration velocity analysis by inverse NMO mainly utilizes only the hyperbolic term. Equation (C-1) provides a way to utilize the non-hyperbolic term to update the velocity ratio, and possibly the anisotropic coefficients as well.

\section{TABLE CAPTIONS}

Table 1: Thomsen (1986) parameters of the five-layer model for evaluating the accuracy of Equation (10)

\begin{tabular}{|l|l|l|l|l|}
\hline Depth $(\mathrm{m})$ & $V_{\mathrm{P} 0}(\mathrm{~m} / \mathrm{s})$ & $V_{\mathrm{S} 0}(\mathrm{~m} / \mathrm{s})$ & $\varepsilon$ & $\delta$ \\
\hline 400 & 1875 & 826 & 0.225 & 0.100 \\
\hline 800 & 2202 & 969 & 0.150 & 0.006 \\
\hline 1200 & 2500 & 1215 & 0.100 & -0.035 \\
\hline 1600 & 3306 & 1819 & 0.134 & 0.000 \\
\hline 2000 & 3368 & 1829 & 0.110 & -0.035 \\
\hline
\end{tabular}




\section{FIGURE CAPTIONS}

Figure 1: A $C$-wave ray path (P down and $\mathrm{S}$ up) in layered VTI media. $x$ is offset,; $V_{\mathrm{P} 0 i}, V_{\mathrm{S} 0 i}$, $\varepsilon_{i}$ and $\delta_{i}$ are interval Thomsen (1986) parameters; $t_{\mathrm{P} 0 i}$ and $t_{\mathrm{S} 0 i}$ are $\mathrm{P}$ - and S-wave vertical oneway interval time.

Figure 2. The offset-dependent behaviour of the $C$-wave moveout signature: (a) A common ACP gather as an input; (b) Hyperbolic moveout correction, ignoring the asymmetry raypath and anisotropy, and only the events at the near offset are flattened; (c) non-hyperbolic moveout correction by equation (1), accounting for the asymmetric raypath but ignoring the anisotropy, and events up to the intermediate offsets are flattened; (d) non-hyperbolic moveout correction using equation (1), accounting for both the asymmetric raypath and anisotropy, and the faroffset events are also satisfactorily flattened.

Figure 3. Results of parameter estimation using equation (1) for the data in Figure 2: (a) velocity analysis for $V_{\mathrm{C} 2}$ over the near offsets $(x / \mathrm{z}=1.5)$, ignoring $\gamma_{\mathrm{eff}}$ and $\chi_{\mathrm{eff}}\left(\gamma_{\mathrm{eff}}=\gamma_{0}=1 ; \chi_{\mathrm{eff}}=0\right)$; (b) $\gamma_{\text {eff }}$ from the intermediate offsets $(\mathrm{x} / \mathrm{z}=2.0)$ with inputs of $V_{\mathrm{C} 2}$ from (a) and $\chi_{\mathrm{eff}}=0$; (3) anisotropic coefficient $\chi_{\text {eff }}$ with inputs of $V_{\mathrm{C} 2}$ and $\gamma_{\text {eff }}$ from (a) and (b), and offsets extended to three times the reflector depth.

Figure 4: $C$-wave scattering from a scatter point in layered VTI media.

Figure 5: Comparison of different methods for calculating the anisotropy parameters: Solid lines - interval model; dotted lines - exact effective values for $\eta_{\text {eff }}$ and $\zeta_{\text {eff }}$ by equation (5), and $\chi_{\text {eff }}$ by equation (4); short dashed lines - approximate values for $\eta_{\text {eff }}$ and $\zeta_{\text {eff }}$ by equation (10).

Figure 6: 2D-4C data example (Courtesy of BG plc): A coarse correlation of the $P$ - and $C$ wave stacked sections for estimating $\gamma_{0}$. The data are from the Lomond field, North Sea .

Figure 7: Interactive analysis for determining $V_{\mathrm{C} 2}, \gamma_{\mathrm{eff}}$ and $\chi_{\mathrm{eff}}$ for the data in Figure 6 . The left panel shows the $V_{\mathrm{C} 2}$ spectrum. The second panel shows $\gamma_{0}$ and $\gamma_{\text {eff. }}$ The third shows $\chi_{\text {eff. }}$ The right panel shows the input gather. 
Figure 8: Updating the migration velocity model using CMP diffraction gathers for the data in Figure 6. (a) A common ACP gather after NMO using the stacking velocity model; (b) the corresponding CIP gather with the initial PSTM model calculated from the stacking model; (c) the CMP diffraction gather obtained by applying inverse NMO to (c); (d) final CIP gather after updating $V_{\mathrm{Cmig}}$, and $\chi_{\text {eff. }}$

Figure 9: Processing results for the data in Figure 6: (a) by the conventional approach of DMO and poststack migration; (b) by the anisotropic PSTM in this paper..

Figure 10. Comparison of the imaging in Figure $9 \mathrm{~b}$ with interpreted horizons from borehole data.

Figure 11. 3D-4C data example (Courtesy of Kerr-McGee North Sea Ltd.): Correlation of Pand $\mathrm{C}$-wave sections to estimate $\gamma_{0}$ from ACP line 050. The left panel is the P-wave section and the right panel is the $\mathrm{C}$-wave section displayed in PP time. The middle panel is the estimated $\gamma_{0}$.

Figure 12. An example of stacking velocity analysis for the 3D-4C data in Figure 11. The left panel shows the $V_{C 2}$ spectrum, and second panel from the left shows the $\gamma_{\text {eff }}$ with a red curve showing $\gamma_{0}$. The blue line indicates the picked values. The third shows $\chi_{\text {eff }}$. The right panel shows the ACP gather.

Figure 13. An example of migration velocity analysis for the data in Figure 11. The left panel shows the hyperbolic spectrum obtained from the CMP diffraction gather. The blue line indicates the picked velocity values. The second panel shows $\gamma_{0}$ and $\gamma_{\text {eff }}$. The third shows $\chi_{\text {eff }}$. The right panel shows the CMP diffraction gather.

Figure 14. The migrated (a) P-wave image and (b) C-image at the location of the middle of two receiver cables for the data in Figure 11. 
26 Li et al.

Figure 15. A comparison between the converted-wave results (displayed in PP time) obtained by the PSTM approach (top figure) and by conventional DMO processing (lower figure) for the data in Figure 11. 


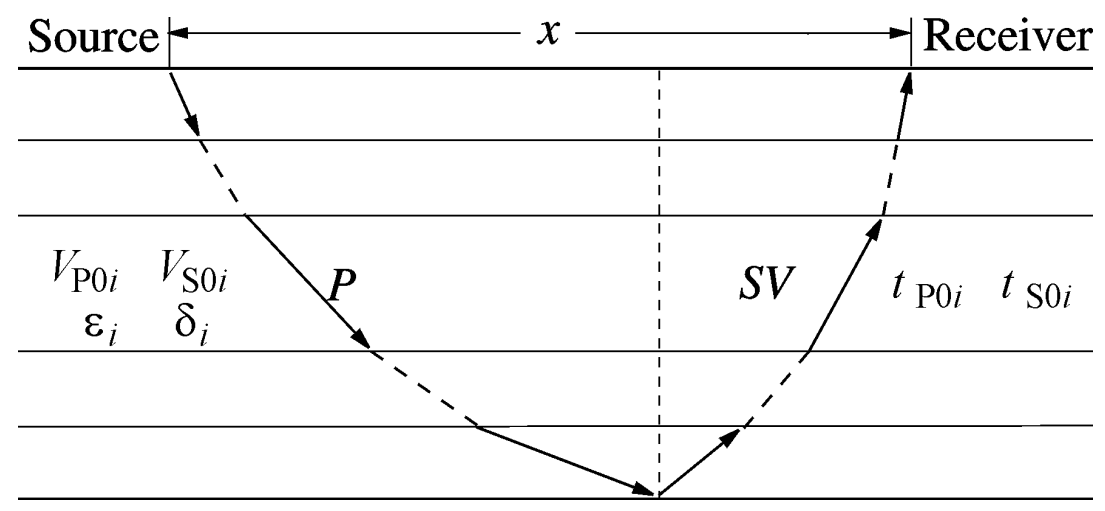

Figure 1: A $C$-wave ray path (P down and $S$ up) in layered VTI media. $x$ is offset,; $V_{\mathrm{P} 0 i}, V_{\mathrm{S} 0 i}, \varepsilon_{i}$ and $\delta_{i}$ are interval Thomsen (1986) parameters; $t_{\mathrm{P} 0 i}$ and $t_{\mathrm{S} 0 i}$ are $\mathrm{P}$ - and $\mathrm{S}$-wave vertical one-way interval time.

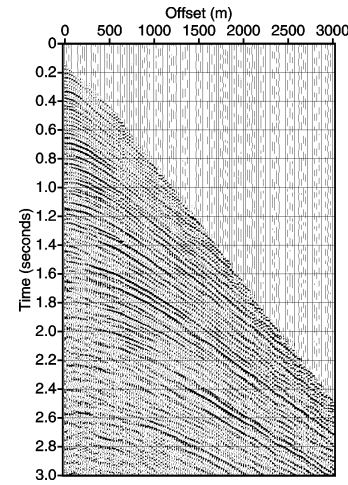

(a)

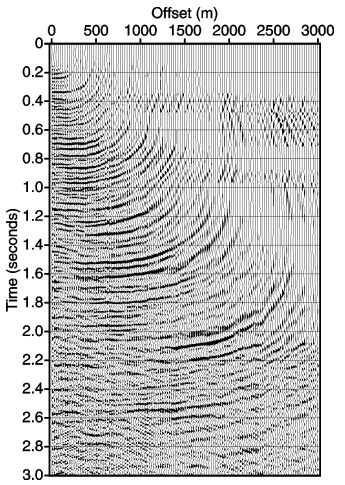

(b)

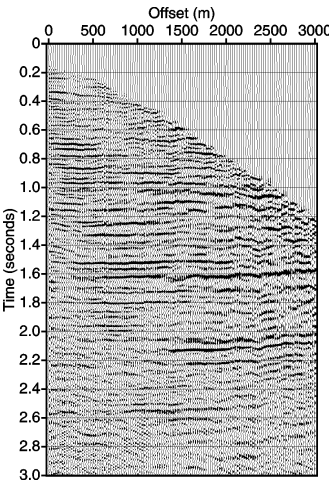

(c)

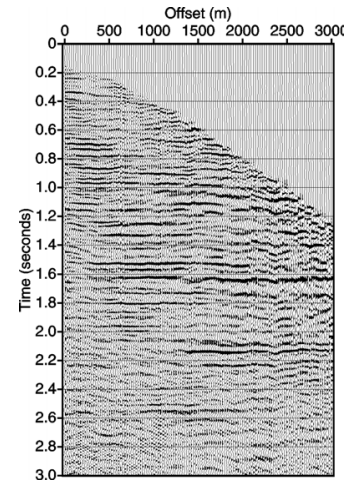

(d)

Figure 2. The offset-dependent behaviour of the $C$-wave moveout signature: (a) A common ACP gather as an input; (b) Hyperbolic moveout correction, ignoring the asymmetry raypath and anisotropy, and only the events at the near offset are flattened; (c) nonhyperbolic moveout correction by equation (1), accounting for the asymmetric raypath but ignoring the anisotropy, and events up to the intermediate offsets are flattened; (d) nonhyperbolic moveout correction using equation (1), accounting for both the asymmetric raypath and anisotropy, and the far-offset events are also satisfactorily flattened. 
$V_{\mathrm{C} 2}$

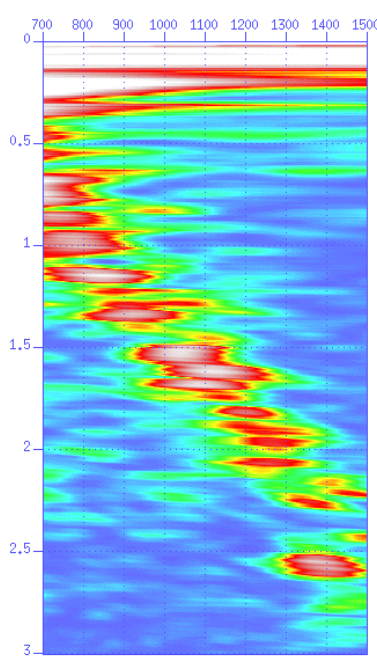

(a)

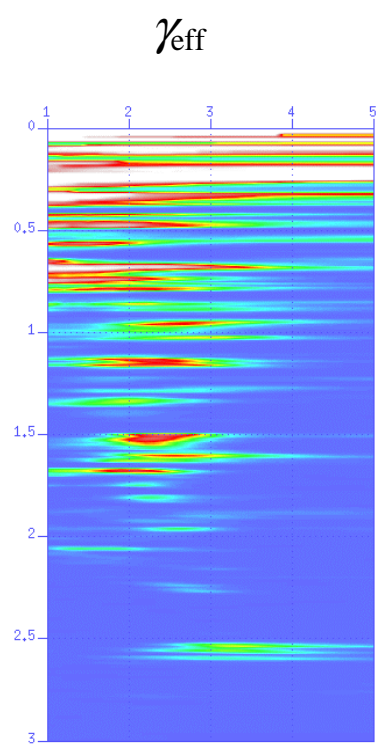

(b)

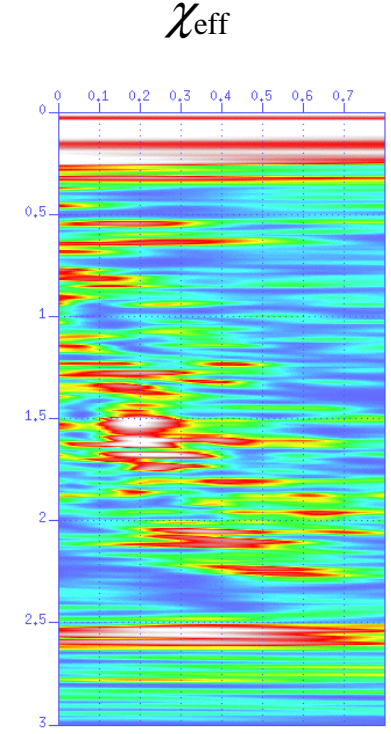

(c)

Figure 3. Results of parameter estimation using equation (1) for the data in Figure 2: (a) velocity analysis for $V_{\mathrm{C} 2}$ over the near offsets $(x / \mathrm{z}=1.5)$, ignoring $\gamma_{\mathrm{eff}}$ and $\chi_{\mathrm{eff}}\left(\gamma_{\mathrm{eff}}=\gamma_{0}=1 ; \chi_{\mathrm{eff}}=0\right)$; (b) $\gamma_{\mathrm{eff}}$ from the intermediate offsets $(\mathrm{x} / \mathrm{z}=2.0)$ with inputs of $V_{\mathrm{C} 2}$ from (a) and $\chi_{\mathrm{eff}}=0 ;(3)$ anisotropic coefficient $\chi_{\text {eff }}$ with inputs of $V_{\mathrm{C} 2}$ and $\gamma_{\text {eff }}$ from (a) and (b), and offsets extended to three times the reflector depth.

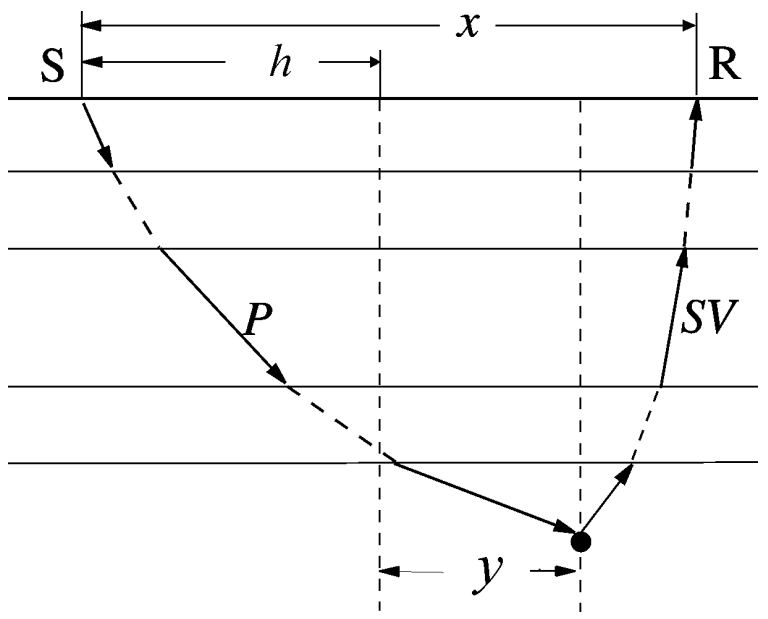

Figure 4: $C$-wave scattering from a scatter point in layered VTI media. 


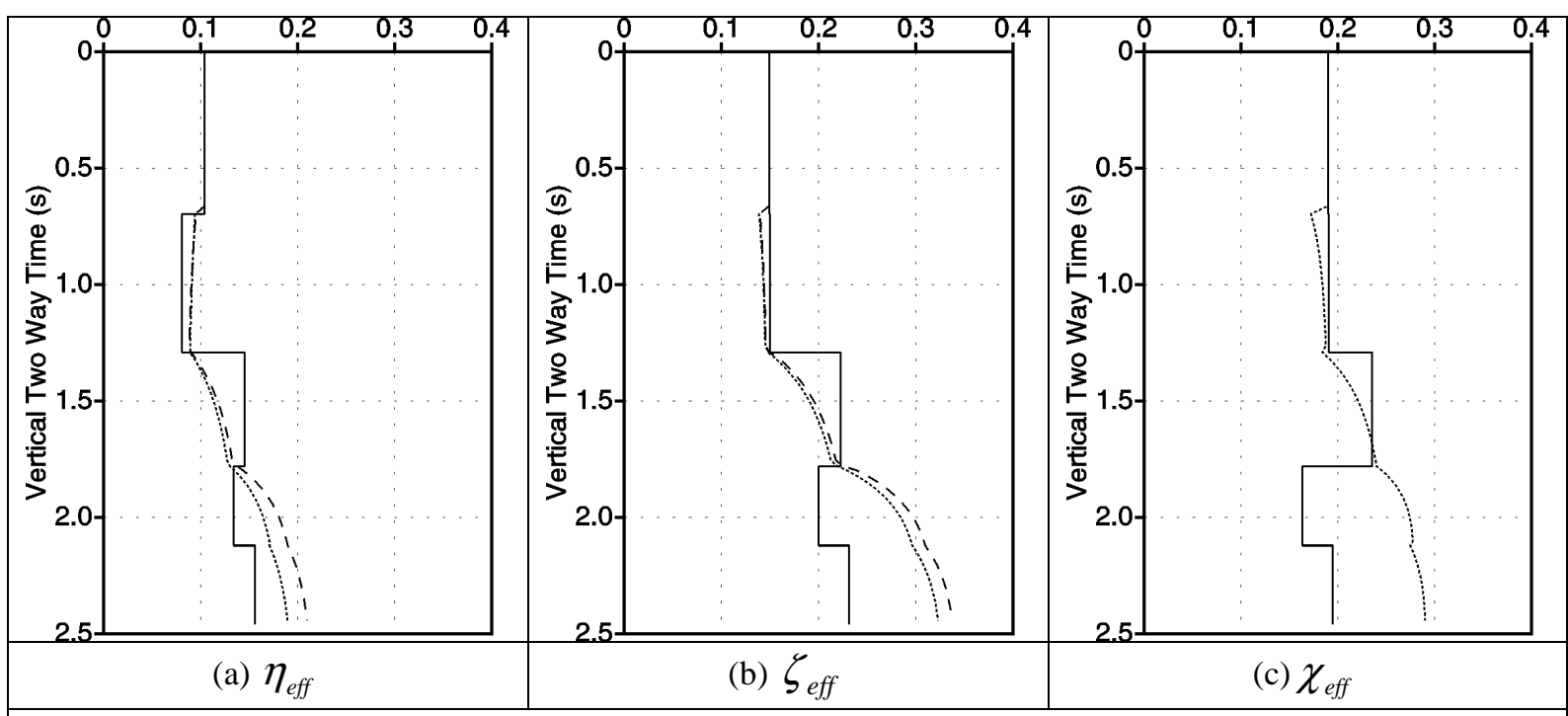

Figure 5: Comparison of different methods for calculating the anisotropy parameters: Solid lines interval model; dotted lines - exact effective values for $\eta_{\text {eff }}$ and $\zeta_{\text {eff }}$ by equation (5), and $\chi_{\text {eff }}$ by equation (4); short dashed lines - approximate values for $\eta_{\text {eff }}$ and $\zeta_{\text {eff }}$ by equation (10).

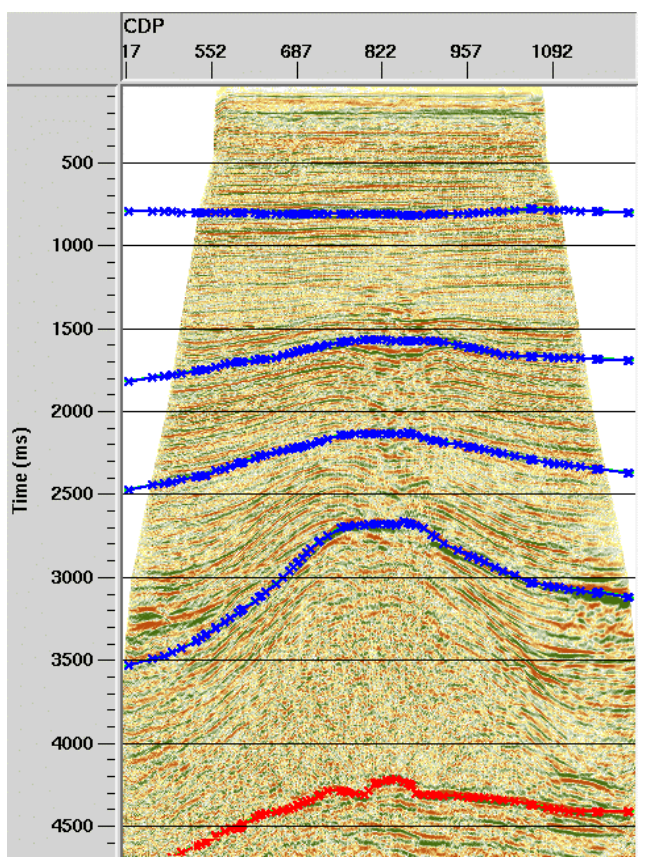

(a) P-wave

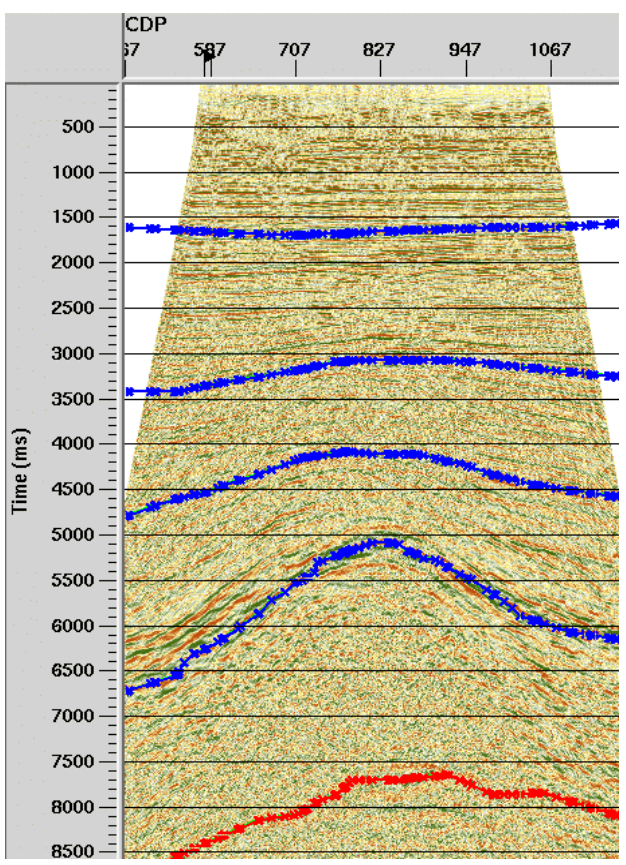

(b) C-wave

Figure 6: 2D-4C data example (Courtesy of BG plc): A coarse correlation of the $P$ and $C$-wave stacked sections for estimating $\gamma_{0}$. The data are from the Lomond field, North Sea. 


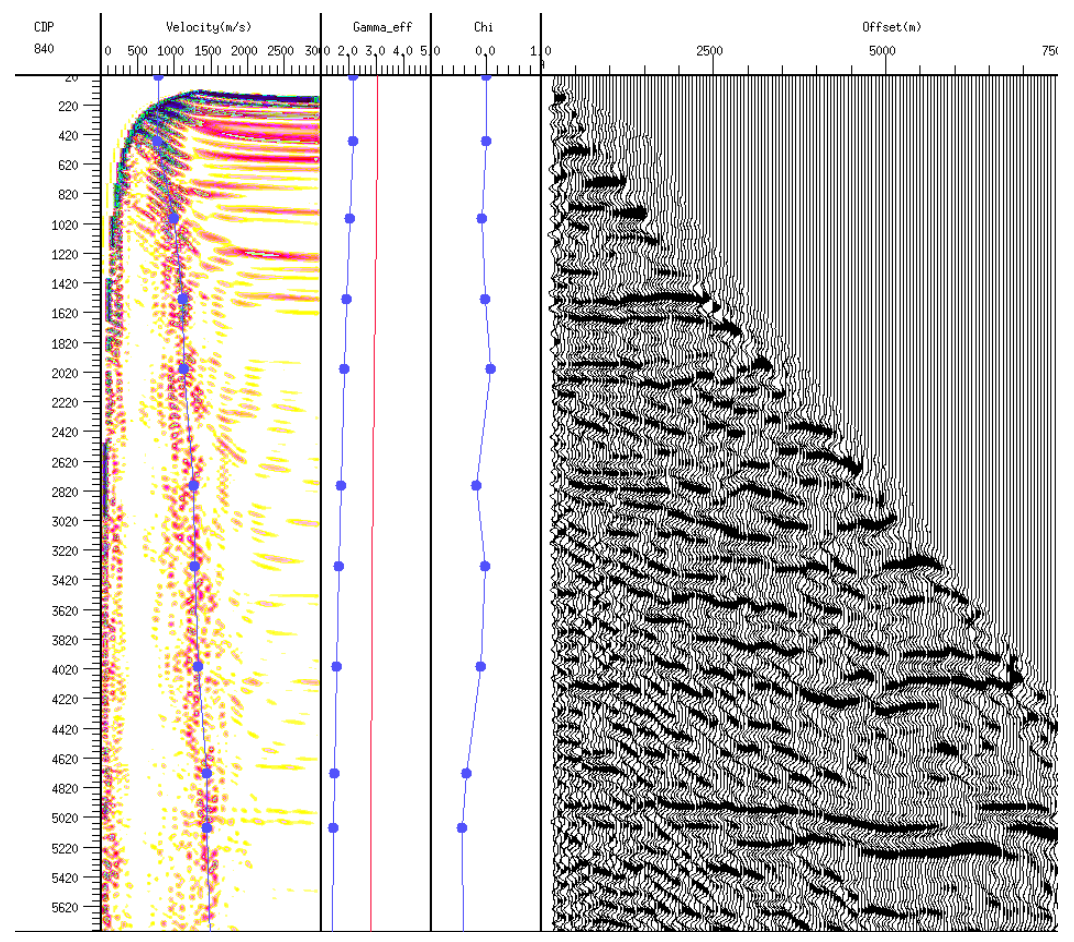

Figure 7: Interactive analysis for determining $V_{\mathrm{C} 2}, \gamma_{\text {eff }}$ and $\chi_{\text {eff }}$ for the data in Figure 6. The left panel shows the $V_{\mathrm{C} 2}$ spectrum. The second panel shows $\gamma_{0}$ and $\gamma_{\text {eff }}$. The third shows $\chi_{\text {eff }}$ The right panel shows the input gather. 


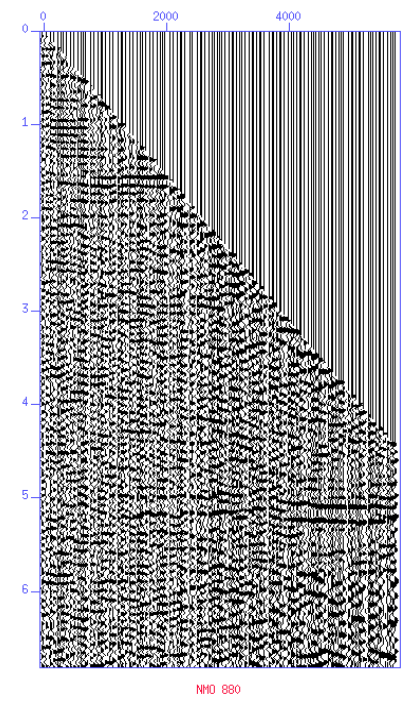

(a) NMO run

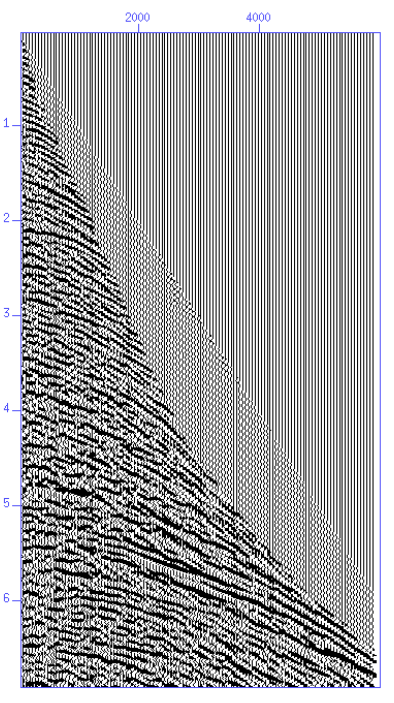

(c) Inverse NMO

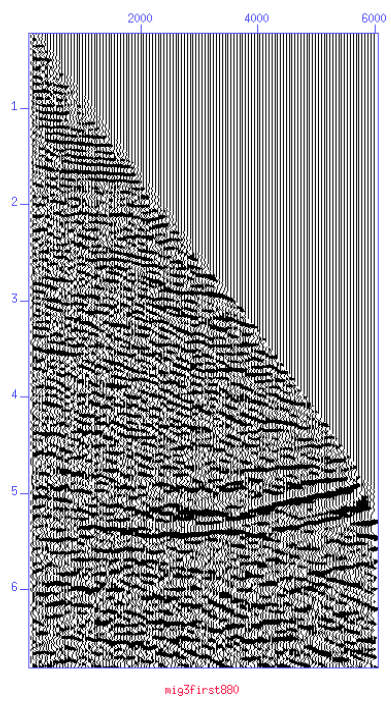

(b) PSTM $1^{\text {st }}$ run

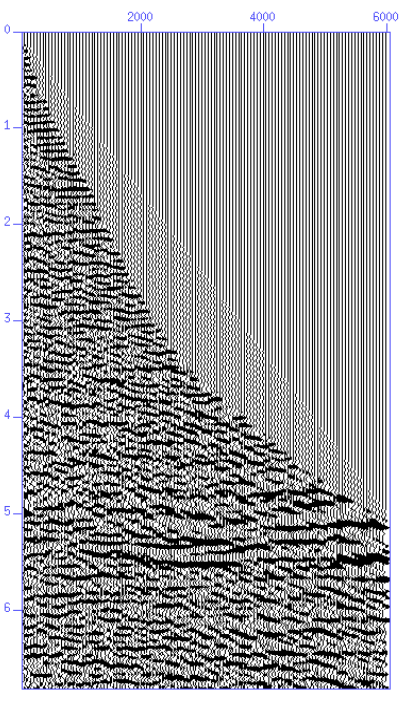

migsfinal1880

(d) ) PSTM $2^{\text {nd }}$ run

Figure 8: Updating the migration velocity model using CMP diffraction gathers for the data in Figure 6. (a) A common ACP gather after NMO using the stacking velocity model; (b) the corresponding CIP gather with the initial PSTM model calculated from the stacking model; (c) the CMP diffraction gather obtained by applying inverse NMO to (c); (d) final CIP gather after updating $V_{\mathrm{Cmig}}$, and $\chi_{\mathrm{eff}}$ 


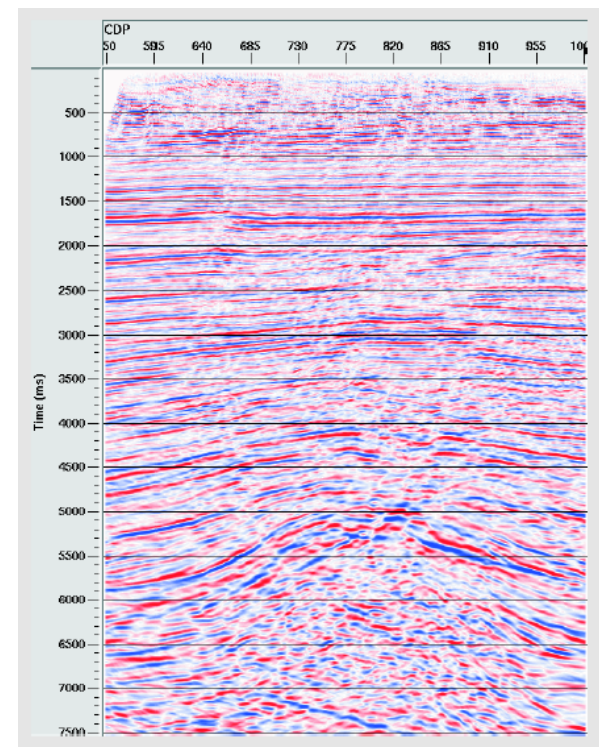

(a) DMO-stack-migration

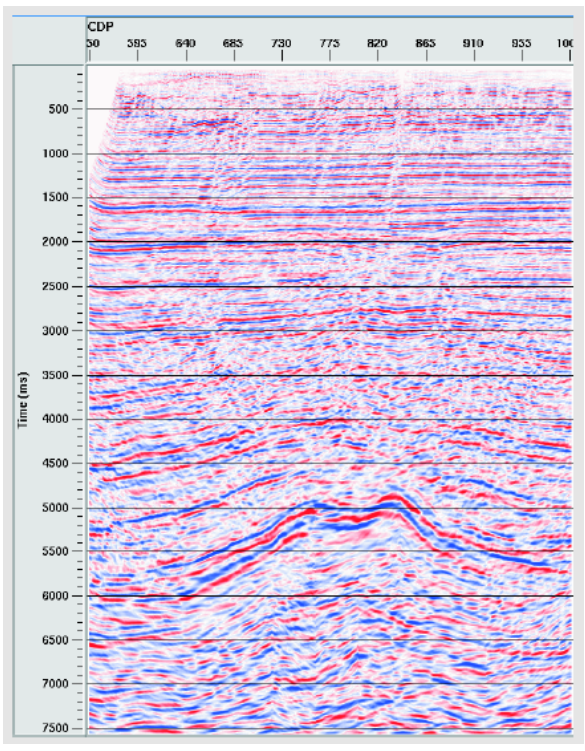

(b) Anisotropic PSTM

Figure 9: Processing results for the data in Figure 6: (a) by the conventional approach of DMO and poststack migration; (b) by the anisotropic PSTM in this paper.

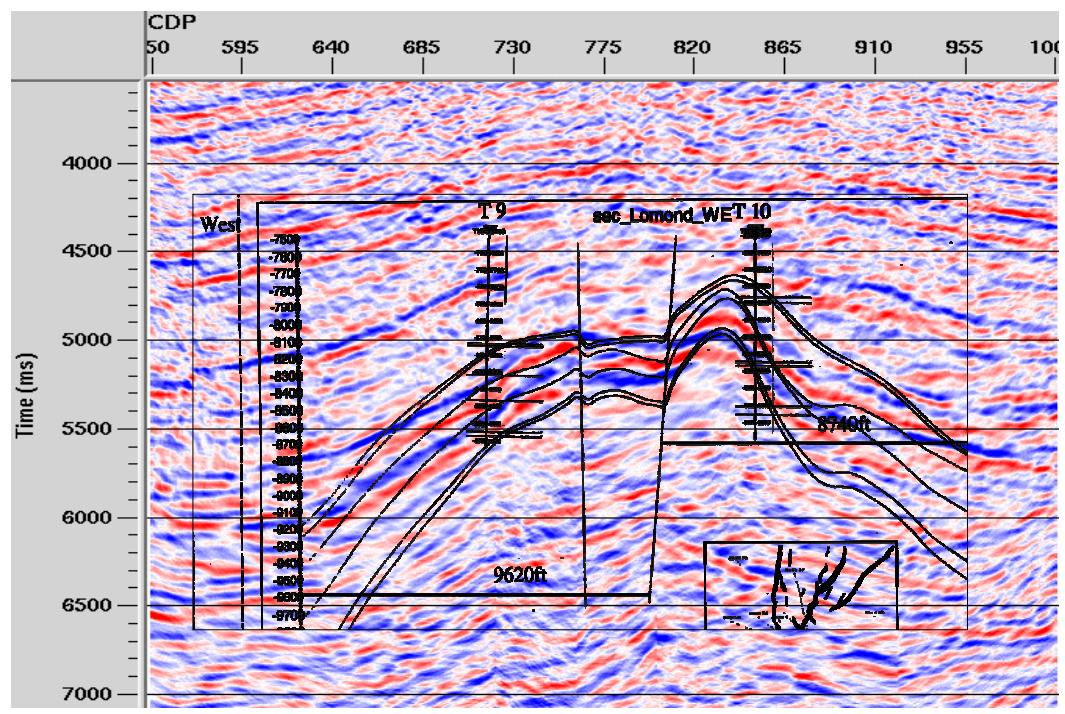

Figure 10. Comparison of the imaging in Figure 9b with interpreted horizons from borehole data. 


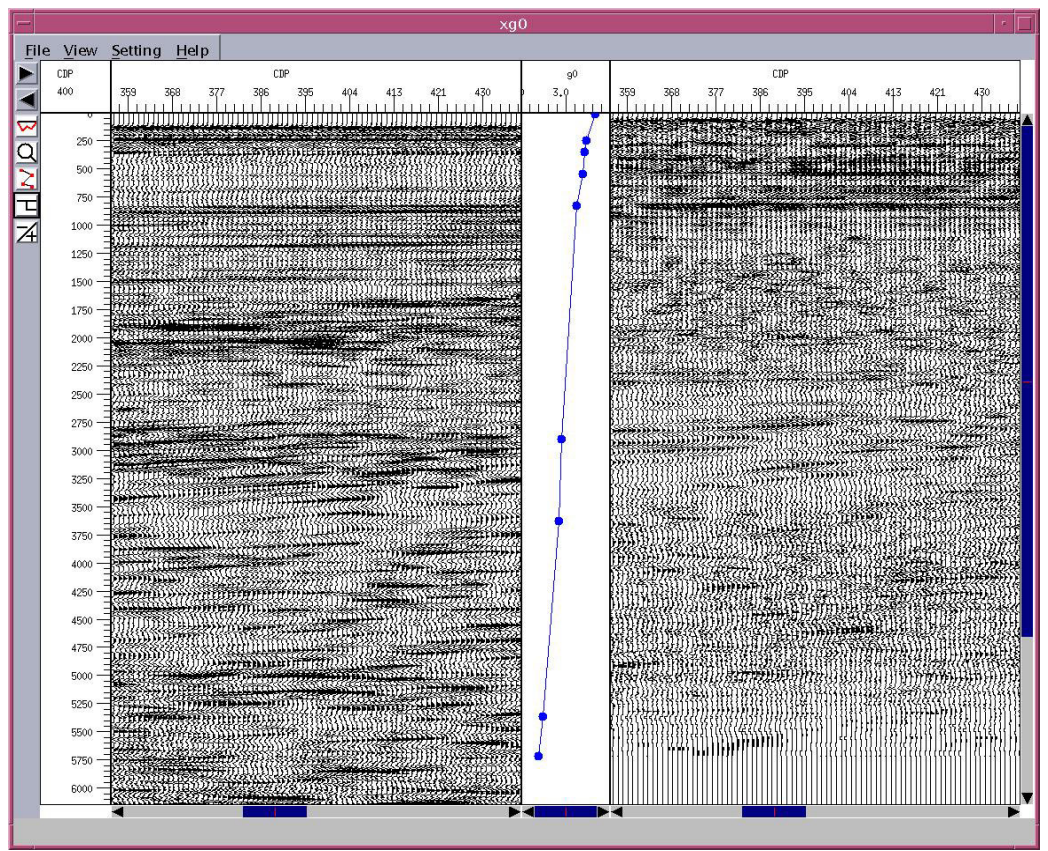

Figure 11. 3D-4C data example (Courtesy of Kerr-McGee North Sea Ltd.) : Correlation of $\mathbf{P}$ - and $\mathbf{C}$-wave sections to estimate $\gamma_{0}$ from ACP line 050. The left panel is the $\mathrm{P}$-wave section and the right panel is the $\mathrm{C}$ wave section displayed in PP time. The middle panel is the estimated $\gamma_{0}$. 


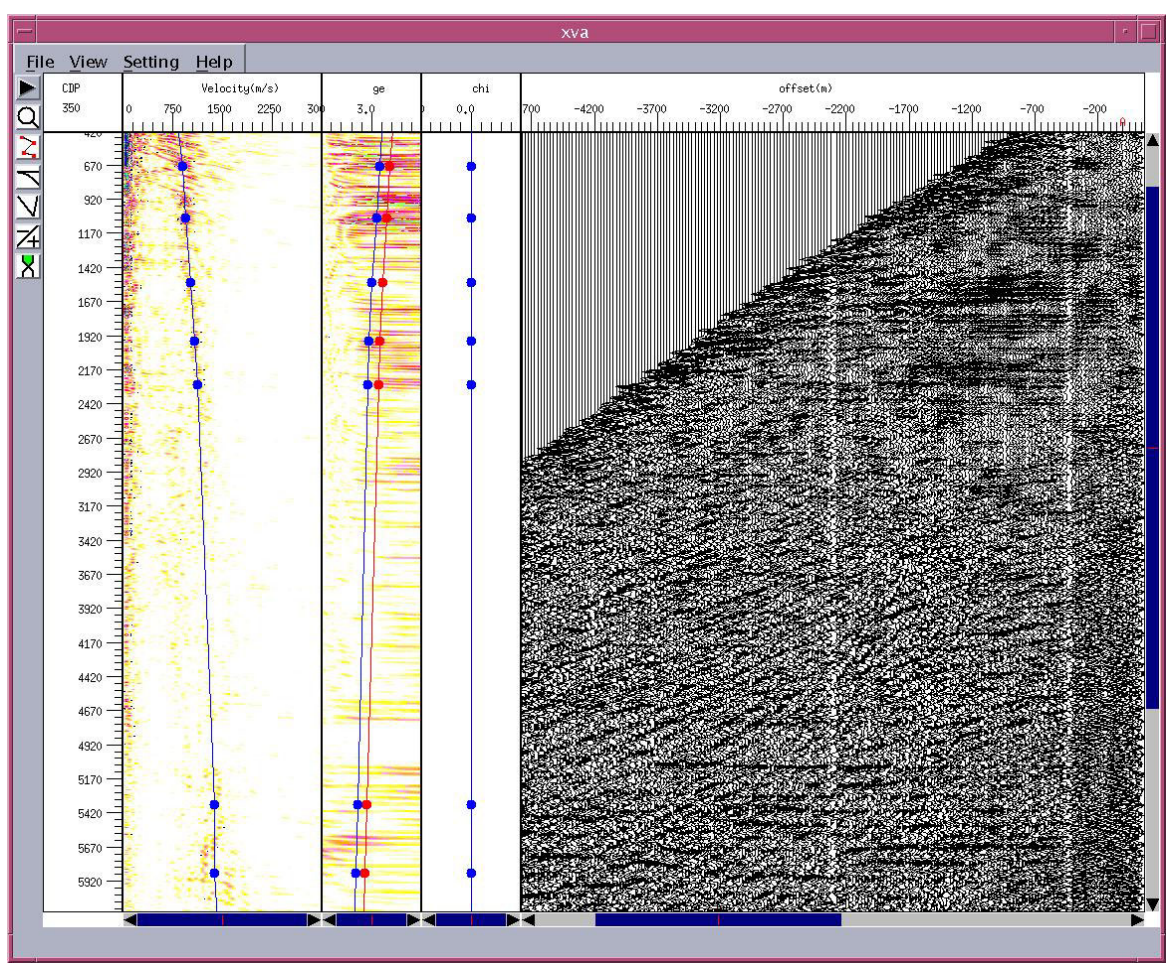

Figure 12. An example of stacking velocity analysis for the data in Figure 11. The left panel shows the $V_{C 2}$ spectrum, and second panel from the left shows the $\gamma_{\text {eff }}$ with a red curve showing $\gamma_{0}$. The blue line indicates the picked values. The third shows $\chi_{\text {eff }}$. The right panel shows the ACP gather. 


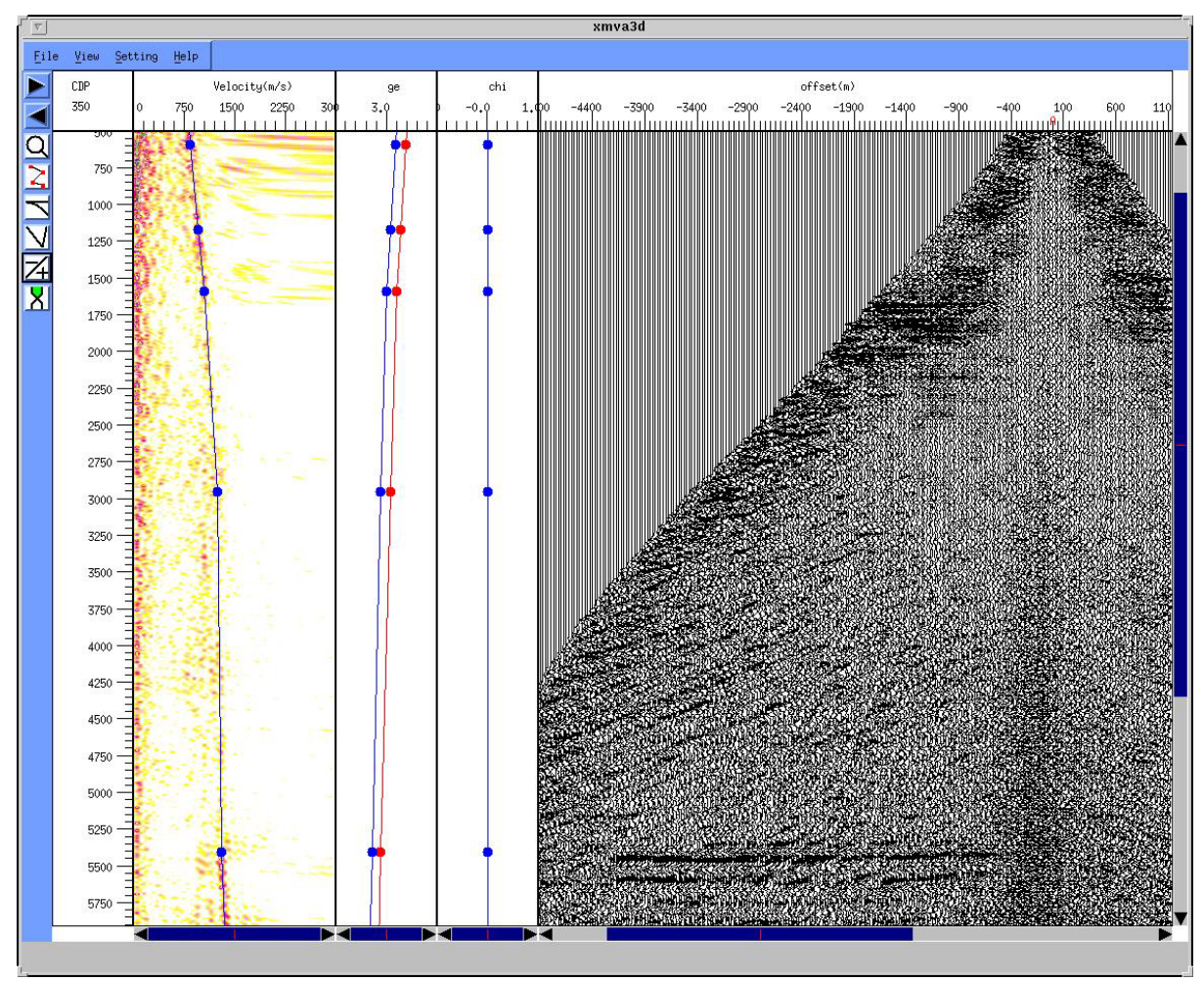

Figure 13. An example of migration velocity analysis for the data in Figure 11. The left panel shows the hyperbolic spectrum obtained from the CMP diffraction gather. The blue line indicates the picked velocity values. The second panel shows $\gamma_{0}$ and $\gamma_{\text {eff }}$. The third shows $\chi_{\text {eff }}$. The right panel shows the CMP diffraction gather. 
36 Li et al.

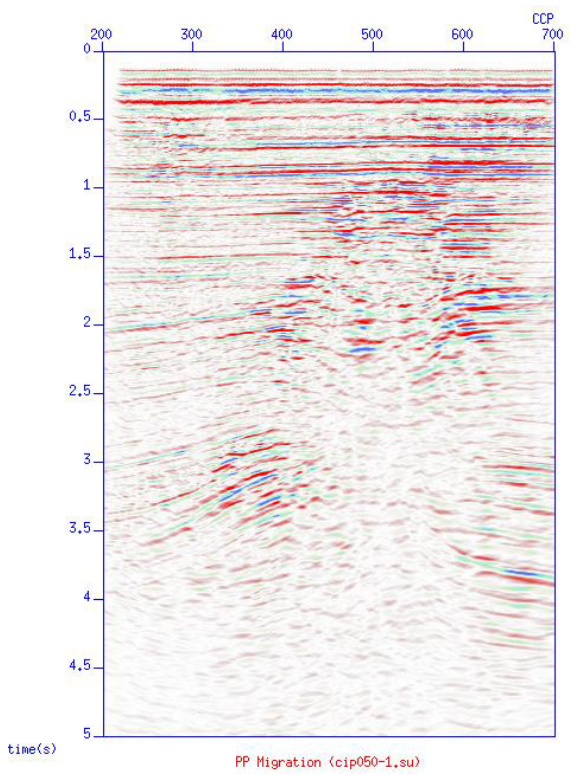

(a) P-wave

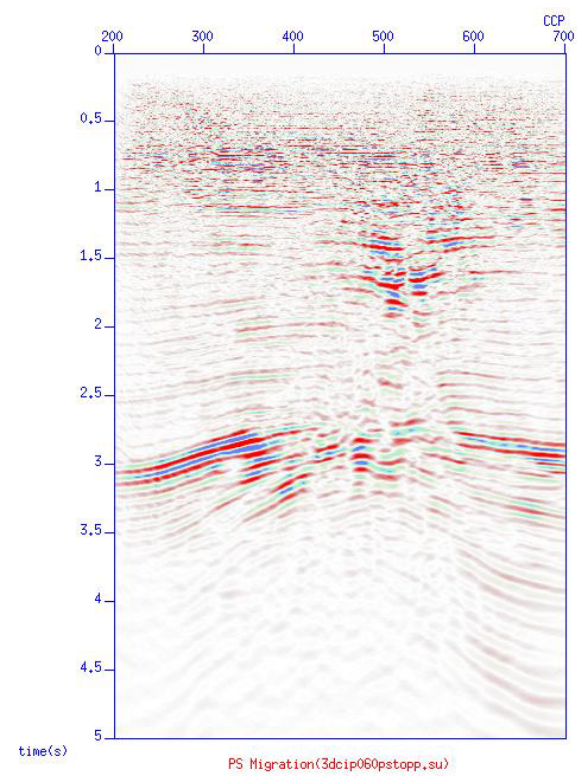

(b) C-wave

Figure 14. The migrated (a) P-wave image and (b) C-image at the location of the middle of two receiver cables for the data in Figure 11.

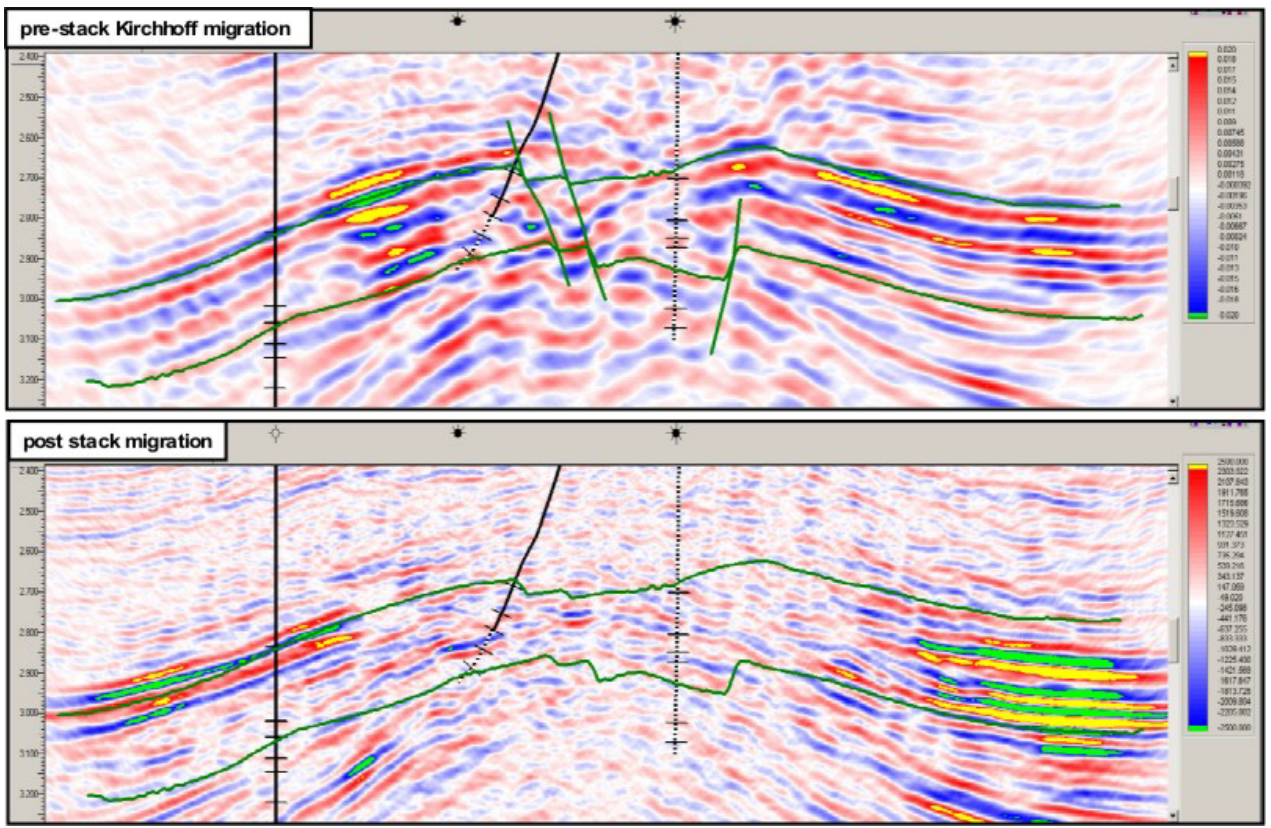

Figure 15. A comparison between the converted-wave results (displayed in PP time) obtained by the PSTM approach (top figure) and by conventional DMO processing (lower figure) for the data in Figure 11. 